\title{
Self-organizing Satellite Constellation in Geostationary Earth Orbit
}

\author{
Garrie S. Mushet, Giorgio Mingotti, Camilla Colombo, and Colin R. McInnes
}

\begin{abstract}
This paper presents a novel solution to the problem of autonomous task allocation for a self-organizing satellite constellation in Earth orbit. The method allows satellites to cluster themselves above targets on the Earth's surface. This is achieved using Coupled Selection Equations (CSE) - a dynamical systems approach to combinatorial optimization whose solution tends asymptotically towards a Boolean matrix describing the pairings of satellites and targets which solves the relevant assignment problems. Satellite maneuvers are actuated by an Artificial Potential Field method which incorporates the CSE output. Three demonstrations of the method's efficacy are given - first with equal numbers of satellites and targets, then with a satellite surplus, including agent failures, and finally with a fractionated constellation. Finally, a large constellation of 100 satellites is simulated to demonstrate the utility of the method in future swarm mission scenarios. The method provides efficient solutions with quick convergence, is robust to satellite failures, and hence appears suitable for distributed, on-board autonomy.
\end{abstract}

\section{Index Terms}

autonomous, constellation, artificial potential, dynamical systems, task allocation

\section{INTRODUCTION}

Recent developments in low-cost spacecraft technologies, and in miniaturization and mass-production, are widening access to space, and are predicted to give rise to future deployments of large satellite constellations. Such constellations offer a number of advantages over single-satellite platforms, including higher temporal resolution, reduced costs and greater coverage, all of which are useful in applications such as Earth observation, space science, and telecommunications.

Additionally, multi-satellite missions based on low-cost technologies offer increased system reliability and robustness, as individual units can be easily replaced in the event of failure, and the longetivity and operational efficacy of any given satellite in the constellation is not critical to the success of the mission.

This work was funded by the European Research Council Advanced Investigator Grant - 227571: VISIONSPACE: Orbital Dynamics at Extremes of Spacecraft Length-Scale. Simulation results were obtained using the EPSRC funded ARCHIE-WeSt High Performance Computer (www.archie-west.ac.uk). EPSRC grant no. EP/K000586/1.

G.S. Mushet, G. Mingotti and C.R. McInnes are with the Department of Mechanical and Aerospace Engineering, University of Strathclyde, Glasgow, G1 1XJ, UK e-mail: (garrie.mushet@strath.ac.uk).

C. Colombo is with the Aerospace Science and Technology Department, Politecnico di Milano, 20156, Italy. 
Traditional satellite constellations have been designed to minimise the number of satellites necessary to meet the coverage requirements of the mission [1], [2]. Existing constellations and those under development, including Globalstar, Iridium, etc [3], [4], [5] , are designed such that the satellites have a fixed relative position, with the propellent budget sized to account only for small, infrequent station-keeping manoeuvres to correct any deviations from those fixed relative positions. This is because the cost of performing significant manoeuvres can be prohibitively high. As a result, much of the literature on autonomous constellation control focuses on station-keeping, and maintaining constant relative positions between constellation members [6], [7], [8], [9], [10], [11].

However, low-cost spacecraft technologies, including high specific impulse low-thrust electric propulsion systems (including pulsed plasma thrusters [12], [13], ion thrusters [14], the University of Michigan's CubeSat Ambipolar Thruster [15], [16], electrostatic colloid thrusters [17], and promising MEMS thrusters [18]) remove the restrictions of traditional constellations, and so the notion of massively distributed constellations of small satellites with the capability of reconfiguring to service real-time changes in coverage demand can be envisioned [19], [20], [21], [22]. This would enable dynamic coverage of specific regions of the Earth's surface. For example, a constellation of telecommunications satellites could respond to localized peaks in demand, which may be transient and unplanned, or may correspond to specific pre-planned events, for example the Olympics, or other large-scale events. Although the lifetime of any individual agent may be short in comparison to existing satellites, this would not affect the reliability of the constellation as a whole, and the constellation could be replenished when necessary. Although launch costs can be significant, as the size and mass of the proposed spacecraft decreases further according to current trends, costs will reduce, and large numbers of satellites could be feasibly launched as a secondary payload.

However, traditional ground-based approaches for control and station-keeping for such large numbers of distributed spacecraft with reconfigurability would prove prohibitively complex and expensive. For lower operational costs and increased system flexibility, autonomy is preferred. Although the implementation of ground-based autonomy would be simpler and less expensive than on-board autonomy, it is limited to Earth applications, as communications delays between ground stations and constellations around other celestial bodies would create a considerably lag between the downlinked sensor measurements, and uplinked control commands. In the interests of generality, on-board autonomy is considered in this work.

Due to the complex nature of the tasks that must be performed by such autonomous constellations, it would be difficult to build autonomy into the system by using, for example, a look-up table of all possible eventualities for task allocation while optimization can be computationally expensive for resource limited small satellites. Instead, it is desirable to follow the example of emergent systems, by implementing behavioural rules which coalesce to produce the desired complex behaviours.

The desired behaviours of an autonomous constellation are many, and include target detection, reconfiguration and manoeuvring, networking, collision avoidance and failure detection. However, one of the first that must be considered is that of task allocation - i.e. in a constellation of many agents, how can it be decided which agents are assigned to which tasks? As the number of agents and tasks increases, the number of possible task assignment combinations grows in a combinatorial explosion, making it difficult for an optimal assignment to be found using 
standard combinatorial optimization processes.

Previous work on task allocation in other multi-agent systems, including unmanned aerial vehicles[23], [24], mobile robots[25], [26], computer systems[27] and wireless sensor networks[28], has focused on developing algorithmic solutions to the relevant assignment problems. Particular works in space-based optimal resource assignment include Radice and McInnes[29], in which behavioural algorithms are developed to address the problem of task prioritisation in autonomous micro-spacecraft. Additionally, in Badawy and McInnes [30], the on-orbit assembly of large space structures is achieved through the use of superquadric potential fields, in which the potential fields are designed such that the systems lowest energy state corresponds to the optimal allocations and optimal trajectories during assembly. Although many of these are effective in the particular scenarios for which they were designed, it can be difficult to validate their performance and behaviour across a wide range of scenarios. In this paper, so-called Coupled Selection Equations (CSEs) are used - these represent a dynamical systems approach to the task allocation problem, and their convergence properties can be validated through simple analysis of the differential equations which govern them. This also allows the Coupled Selection differential equations to be embedded within the state space representation of the multi-agent system, providing an elegant link between individual agent dynamics, and the constellation's task assignment operations. They also offer the possibility of elegantly dealing with the extension of conventional swarming concepts to those in which the swarm members have different capabilities and/or equipment. This is particularly useful in the domain of micro-spacecraft, where spacecraft subsystems can be fractionated across multiple agents.

CSEs have been successfully implemented in a variety of multi-agent scenarios. Lafrenz et. al implemented CSEs to assign the roles of attacker, defender and goal-keeper among a team of soccer playing robots, by constructing utility and activation parameters[31]. In the realm of space engineering, Molnar developed CSEs as a method for assigning tasks to self-organising space colonies, in which various desirable characteristics of the method were demonstrated including automatic activation of spare agents, fault tolerance and its utility in distributed sensor networks[32]. Starke et. al utilised CSEs to assign subtasks to robots in the self-organised assembly of space station components[33], deciding which agents were to retrieve and assemble the various components. Multiple other examples are found in literature, including the autonomisation of manufacturing processes performed by mobile robots[34], [35]. However, the implementation and the analysis of the method in a highly reconfigurable satellite constellation has not been previously investigated.

Hence, this paper presents a novel dynamical systems approach to the problem of task allocation in a satellite constellation for re-allocating satellite resources to match demand on the Earth's surface. A single ring of satellites at geostationary earth orbit (GEO) is considered for ease of illustration. The method implements CSEs to solve the linear two-index and axial three-index assignment problems. These represent the problems of assigning tasks to agents from a homogenous constellation of satellites, and from a fractionated constellation containing two different satellite types, respectively. A fractionated constellation could represent one in which the tasks related to payload, power and other subsystems are disaggregated across various satellites. The solutions delivered by the Coupled Selection Equations represent near-optimal pairings of satellites to targets on the Earth's surface, with the manoeuvres 
being actuated by a simple artificial potential function.

The remainder of the paper is organized as follows. The next section introduces the constellation model - a single circular ring of $n$ spacecraft at GEO. The task allocation problem is then introduced. Coupled Selection Equations are then proposed as a solution to the task allocation problem. The results of the Coupled Selection Equations for three simple demonstration cases of the method are then given, followed by the manoeuvring strategy for the satellites. A summary of the various elements of the proposed control scheme is then given. The method is then applied to a large constellation of 100 satellites to show the efficacy of the method in future swarm mission scenarios. Finally, extensions of the method are discussed and conclusions are made.

\section{Constellation Model}

The constellation is modelled as a ring of $n$ satellite members, initially at geostationary altitude, and azimuthally equispaced, as described by (1).

$$
\left.\begin{array}{c}
r_{j_{0}}=r_{\mathrm{GEO}} \\
\theta_{j_{0}}=\frac{2 \pi(i-1)}{n}
\end{array}\right\} \quad(j=1, \cdots, n)
$$

where $r_{j_{0}}$ and $\theta_{j_{0}}$ are the initial orbit radius and true anomaly of the $j$ th satellite, $n$ is the number of satellites on the constellation, and $r_{\mathrm{GEO}}$ is the orbit radius for GEO, equal to $42,164.1 \mathrm{~km}$.

The orbit of each satellite can be propagated according to the Modified Equinoctial Elements elements given in (2) [36], and which have been chosen in order to avoid singularities associated with the standard Gaussian form of the Keplerian elements in the $0^{\circ}$ inclination circular orbits employed in this paper.

$$
\begin{aligned}
& \dot{p}=\frac{2 p}{w} \sqrt{\frac{p}{\mu}}\left[\begin{array}{lll}
a_{r} & a_{t} & a_{n}
\end{array}\right]\left[\begin{array}{lll}
0 & 1 & 0
\end{array}\right]^{T} \\
& \dot{f}=\sqrt{\frac{p}{\mu}} \frac{1}{w}\left[\begin{array}{lll}
a_{r} & a_{t} & a_{n}
\end{array}\right]\left[\begin{array}{lll}
w \sin L & (w+1) \cos L+f & -(h \sin L-k \cos L) g
\end{array}\right]^{T} \\
& \dot{g}=\sqrt{\frac{p}{\mu} \frac{1}{w}}\left[\begin{array}{lll}
a_{r} & a_{t} & a_{n}
\end{array}\right]\left[\begin{array}{lll}
-w \cos L & (w+1) \sin L+g & (h \sin L-k \cos L) f
\end{array}\right]^{T} \\
& \dot{h}=\sqrt{\frac{p}{\mu}} \frac{s^{2}}{2 w}\left[\begin{array}{lll}
a_{r} & a_{t} & a_{n}
\end{array}\right]\left[\begin{array}{lll}
0 & 0 & \cos L
\end{array}\right]^{T} \\
& \dot{k}=\sqrt{\frac{p}{\mu}} \frac{s^{2}}{2 w}\left[\begin{array}{lll}
a_{r} & a_{t} & a_{n}
\end{array}\right]\left[\begin{array}{lll}
0 & 0 & \sin L
\end{array}\right]^{T} \\
& \dot{L}=\sqrt{\mu p}\left(\frac{w}{p}\right)^{2}+\sqrt{\frac{p}{\mu}} \frac{1}{w}\left[\begin{array}{lll}
a_{r} & a_{t} & a_{n}
\end{array}\right]\left[\begin{array}{lll}
0 & 0 & (h \sin L-k \cos L)
\end{array}\right]^{T}
\end{aligned}
$$

where $\mu$ is the standard gravitational parameter of Earth, $w=1+f \cos L+g \sin L, s^{2}=1+h^{2}+k^{2},\left[\begin{array}{ll}a_{r} & a_{t} \\ a_{n}\end{array}\right]$ is the acceleration vector consisting of radial, tangential and out-of-plane accelerations respectively, and $p, f, g, h$, $k$ and $L$ are the modified equinoctial elements which are related to the standard Keplerian elements via (3). 


$$
\begin{aligned}
& p=a(1-e)^{2} \\
& f=e \cos (\omega+\Omega) \\
& g=e \sin (\omega+\Omega) \\
& h=\tan i / 2 \cos \Omega \\
& k=\tan i / 2 \sin \Omega \\
& L=\Omega+\omega+\nu
\end{aligned}
$$

where $a$ is the semi-major axis, $e$ is eccentricity, $i$ is inclination, $\omega$ is argument of perigee, $\Omega$ is right ascension of the ascending node, and $\nu$ is the true anomaly.

It is now assumed that the spacecraft have the ability to thrust in the tangential direction only, that the satellite orbits remain quasi-circular, so that $p \approx r$ where $r$ is the orbital radius, and $L \approx \nu$ throughout the manoeuvres, and that there will be no out-of-plane motion, so $\dot{h}=\dot{k} \approx 0$.

Applying these assumptions, the equations of motion simplify to those given in (4), where the $j$ notation is re-introduced to describe the multiple satellite system, and is understood to run from 1 to $n$.

$$
\begin{aligned}
\dot{r}_{j} & =2 \sqrt{\frac{r_{j}^{3}}{\mu}} a_{t j} \\
\dot{\nu}_{j} & =\sqrt{\frac{\mu}{r_{j}^{3}}}
\end{aligned}
$$

It has been previously shown that, for small maneuvers of the type simulated in this work, the eccentricity of a satellite orbit remains small, hence justifying the assumption that the satellite orbits remain quasi-circular [37].

For numerical propagation a Runge-Kutta method with a relative tolerance of $10^{-3}$, absolute tolerance of $10^{-6}$ and a maximum step size of $100 \mathrm{~s}$ has been employed.

The analytical solution to (4) for constant acceleration can be obtained and was used in the development of the controller later in Section V, such that

$$
\begin{aligned}
r_{j}(t) & =\frac{r_{0 j}}{\left(1-a_{t j} \sqrt{\frac{r_{0 j}}{\mu}} t\right)^{2}} \\
\theta_{j}(t) & =-\frac{\mu}{4 a_{t j} r_{0 j}^{2}}\left(\left(a_{t j} \sqrt{\frac{r_{0 j}}{\mu}} t-1\right)^{4}-1\right)+\theta_{0 j} .
\end{aligned}
$$

Two different constellation types are investigated in the test cases of this paper - one in which the constellation consists of $n$ homogenous satellites, and a fractionated constellation in which the constellation consists of $n_{1}$ satellites of type 1 , and $n_{2}$ satellites of type 2 . In both cases, the initial spacing and dynamics of each satellite are the same as described above. 


\section{TASK Allocation Problem}

The task allocation problem, in this context, is the problem of deciding which satellites on the constellation should be assigned to various targets of interest on the Earth's surface.

In a scenario where the constellation consists of homogenous satellites, the task allocation problem can be described by the linear two-index assignment problem. When the constellation is fractionated and consists of two different types of satellite with different capabilities, and one of each satellite type must visit each target, the problem can be described by the axial three-index assignment problem [38].

\section{A. Linear Two-Index Assignment Problem}

The linear two-index assignment problem is to minimise the total costs, $c$, associated with the pairings between $N=1, \ldots, n$ targets and satellites, and is stated compactly in (6) - (8).

$$
\begin{array}{ll}
\underset{X_{i j}}{\operatorname{minimise}} & c=\sum_{i, j} C_{i j} X_{i j} \\
\text { subject to } & \sum_{i} X_{i j}=1, \quad \forall j \in N \\
& \sum_{j} X_{i j}=1, \quad \forall i \in N
\end{array}
$$

Here $C_{i j}$ is the cost of pairing target $i$ and satellite $j$, and $X_{i j}$ is a Boolean variable describing the status of the pairing, and is defined in (9).

$$
X_{i j}=\left\{\begin{array}{cc}
1 & \text { if satellite } j \text { is to visit target } i \\
0 & \text { otherwise }
\end{array}\right.
$$

The constraints of (7) - (8) represent the requirement that each target can be visited by only one satellite, and each satellite can only visit one target, respectively. This implies that $\mathbf{X}=\left[X_{i j}\right]$ must be a permutation matrix. It should be noted that if it is desired for a target to be visited by multiple satellites, that target will simply be replicated within the cost and Boolean matrices as many times as the number of satellites it requires.

In this paper, the classic two-index assignment problem as described above was transformed to the equivalent maximization problem, where the winnings, $W_{i j}$ associated with each pairing are to be maximized, and the winnings are related to the costs through the linear transformation given in (10).

$$
W_{i j}=-\gamma \cdot C_{i j}+\delta, \quad \gamma>0, \quad \gamma, \delta \in \mathbb{R}, \forall(i, j) \in N^{2}
$$

where $\gamma$ and $\delta$ are arbitrary constants.

This transformation from a problem of minimization of costs to a problem of maximization of winnings is made due to the nature of Coupled Selection Equations, which are only suitable for solving maximization problems. 


\section{B. Axial Three-Index Assignment Problem}

The axial three-index assignment problem is an extension to the linear two-index assignment problem. In this work, it is used to represent the problem of assigning satellites to targets on a fractionated constellation where two different types of satellites exist, and each target must be visited by a satellite of each type. This could represent a constellation of disaggregated spacecraft, where payload, power and other subsystems are distributed across separate agents and each satellite type has a different specialization. If we have $N=1, \ldots, n$ targets, and $N=1, \ldots, n$ of satellites of types 1 and 2, then the problem is stated compactly in (11) - (14).

$$
\begin{array}{ll}
\underset{X_{i j k}}{\operatorname{minimise}} & c=\sum_{i, j, k} C_{i j k} X_{i j k} \\
\text { subject to } & \sum_{i} X_{i j k}=1, \quad \forall(j, k) \in N^{2} \\
& \sum_{j} X_{i j k}=1, \quad \forall(i, k) \in N^{2} \\
& \sum_{k} X_{i j k}=1, \quad \forall(i, j) \in N^{2}
\end{array}
$$

Then, the Boolean variable is defined according to (15).

$$
X_{i j k}=\left\{\begin{array}{lc}
1 & \text { if satellites } j \& k \text { are to visit target } i \\
0 & \text { otherwise }
\end{array}\right.
$$

Again, the problem is transformed to a maximization problem in this work using the transformation in (10).

\section{Coupled Selection Equations}

A selection equation is defined as a dynamical system in which various modes of the system are set in competition, and only one mode will survive, whilst all others converge to zero.

In the most basic scenarios, the behaviour may seem trivial, however in more complex situations the coupled selection equations provide efficient solutions in spite of often complex interactions between the modes.

Because of this behaviour, the method lends itself well to providing solutions for the task allocation problem. The most basic of these selection equations is given in (16).

$$
\dot{\xi}_{i}=\xi_{i}\left(1-\xi_{i}^{2}-\beta \sum_{i^{\prime} \neq i} \xi_{i^{\prime}}^{2}\right)
$$




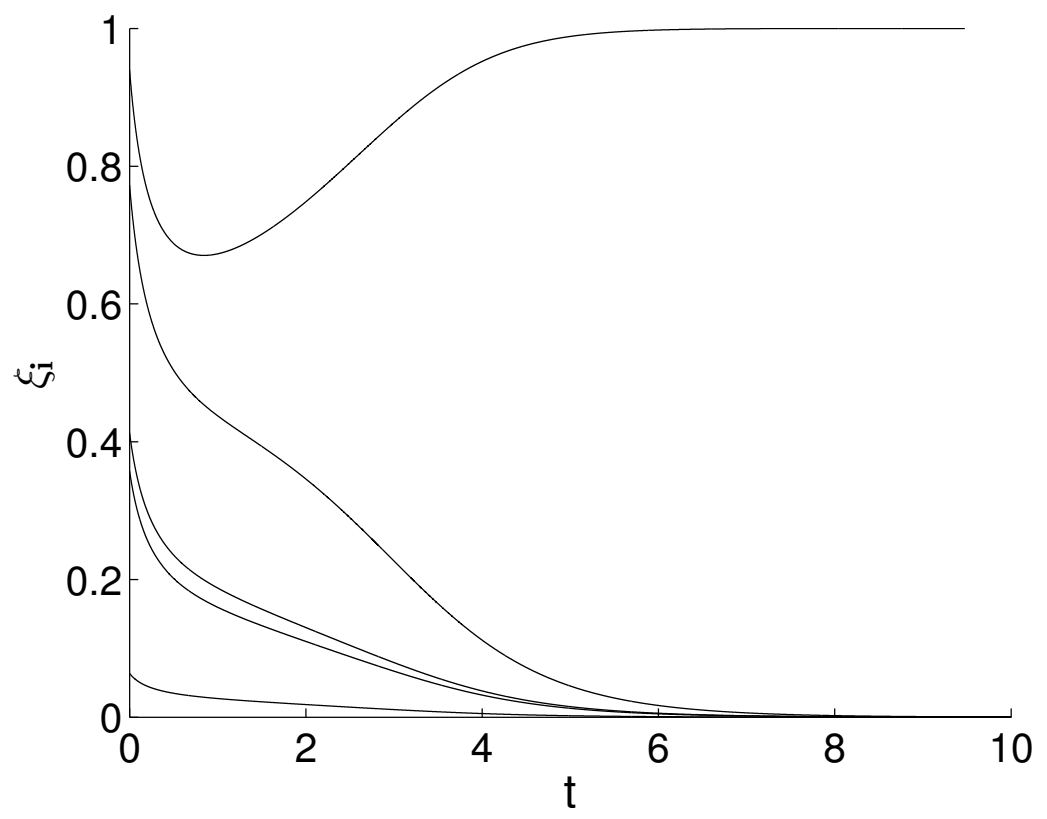

Fig. 1. Typical time evolution of single-index Coupled Selection Equations.

where $\xi_{i}$ is the value of the $i$ th coupled selection mode.

It can be shown that the stable equilibrium of (16) corresponds to a situation in which the $\xi_{i}$ with the highest initial value converges to 1 , while all others converge to 0 , subject to the conditions that $\xi_{i}(0) \geq 0, \forall i$ and $\beta>1$, where $\beta$ is a parameter which determines the influence of the modes on each other's time evolution.

A simple demonstration of the time evolution of this system is shown in Fig. 1 where 5 modes are initialized.

This equation can be extended for use in the linear two-index assignment problem by numbering the variables with two indices and extending the coupling terms as given by (17).

$$
\dot{\xi}_{i j}=\xi_{i j}\left(1-\xi_{i j}^{2}-\beta\left(\sum_{i^{\prime} \neq i} \xi_{i^{\prime} j}^{2}+\sum_{j^{\prime} \neq j} \xi_{i j^{\prime}}^{2}\right)\right)
$$

where $\xi_{i j}$ is the coupled selection mode describing the status of the pairing between target $i$ and satellite $j$, and $\beta$ is a scaling parameter.

When the matrix of coupled selection variables, $\boldsymbol{\Xi}=\left[\xi_{i j}\right]$, is initialized with the non-negative winnings from the linear two-index assignment problem, where each winning from the winnings matrix, $\mathbf{W}=\left[W_{i j}\right]$, is transformed from the corresponding costs, $\mathbf{C}=\left[C_{i j}\right]$, using (10), where $\gamma=(\max \mathbf{C})^{-1}$ and $\delta=1$ and have been set such that $W_{i, j} \in[0,1], \forall i, j$, then it can be shown that in the limit, the coupled selection variables tend asymptotically towards a permutation matrix which solves the linear two-index assignment problem, $\hat{\mathbf{X}}$. This process is formalized in (18) [39]. The criteria for globally optimal solutions to the assignment problem is detailed in [38]. It is also a requirement that $\beta>\frac{1}{2}$ (and, in general $\beta>\frac{1}{D}$ where $D$ is the dimension of the coupled selection variables). The series of transformations are defined by (18). 


$$
\mathbf{C} \mapsto \mathbf{W} \mapsto \boldsymbol{\Xi}(0) \rightarrow \lim _{t \rightarrow \infty} \boldsymbol{\Xi}(t)=\hat{\mathbf{X}}
$$

In this application, the costs are chosen to be equal to the normalized sum of the angular distance, $\phi_{i j}$, between the targets and satellites and the $\Delta v$ already expended by each satellite, as given by (19).

$$
C_{i j}=\kappa \phi_{i j}+\tau \Delta v_{j}, \quad-\pi \leq \phi_{i j}<\pi, \quad \kappa, \tau \in \mathbb{R}
$$

where $\phi_{i j}$ is the phasing angle between target $j$ and satellite $i, \Delta v_{j}$ is the cumulative expenditure of $\Delta v$ for satellite $j$, and $\kappa$ and $\tau$ are weighting parameters.

This is to ensure that the selection equations allocate satellites according to their angular distance from targets, in order to minimize maneuver time, and also that the workload is balanced across the constellation.

It should be noted that, in large constellations, the most appropriate allocations are not necessarily obvious, and the Coupled Selection Equations provide efficient allocations in these situations.

It should also be noted that while the value of $\phi_{i j}$ is limited to $[-\pi, \pi]$, the value of $\Delta v_{j}$ is limited only by the maximum fuel load carried by each satellite. Hence, after some time, the $\Delta v_{j}$ term will come to become more important in the cost equation, forcing the allocations to be chosen such that the workload across the constellation is balanced in the long run, as satellite resourced deplete.

One further benefit of the two-index Coupled Selection Equations that makes them appropriate for this application is that they will still converge to a Boolean matrix if they are modified to represent the case where there is a surplus of satellites in comparison to targets. In this case, the constraints of (6) - (8) will not be met, but it will still be the case that each target will be visited by one satellite only, and that the satellites will be assigned in such a way that (6) will still be minimized.

The coupled selection equation is extended further for use in the axial three-index assignment problem. Again, another index is added to the variable numbering, and more coupling terms are added as appropriate as given by (20).

$$
\dot{\xi}_{i j k}=\xi_{i j k}\left(1+(3 \beta-1) \xi_{i j k}^{2}-\beta\left(\sum_{i^{\prime}} \xi_{i^{\prime} j k}^{2}+\sum_{j^{\prime}} \xi_{i j^{\prime} k}^{2}+\sum_{k^{\prime}} \xi_{i j k^{\prime}}^{2}\right)\right)
$$

Equation 20 will converge according to (18) so long as $\beta>\frac{1}{3}$ and $\xi_{i j k}(0)>0, \forall i, j, k$. In this case, each cost $C_{i j k}$ is set equal to the sum of the angular distances between satellite $i$ and target $k$ and satellite $j$ and target $k$.

Although the asymptotically stable solutions of Eqs. 16, 17 and 20 correspond to valid solutions, there may be some unstable equilibria - hence, noise is added to the equations if the time derivatives fall below a set tolerance, in order to prevent stagnation close to unsuitable unstable equilibria.

Because the equations can be shown to be gradient flows with asymptotically stable equilibria and containing no more complicated attractors than this, a simple first order forward Euler method integrator is sufficient for numerically propagating the equations. This has further advantages for implementation on a highly distributed 
constellation of small satellites, as it does not require significant on-board processing from each agent in order for them to propagate the equations on-orbit [38]. The concept of on-orbit distributed computing in small-satellite constellations has recently been explored by Gunter \& Maessen and deemed feasible, lending support to the notion that easily integratable systems of equations such as Coupled Selection Equations would be amenable to such implementations[40].

Below, three demonstration cases of the Coupled Selection Equations solving constellation task allocation problems are given, with each focusing on a different aspect of the coupled selection equations.

\section{A. Demonstration of CSEs for Equal Number of Satellites and Targets}

In this demonstration, a constellation of six homogenous satellites are initially equi-spaced on geostationary orbit with an initial $\Delta v$ expenditure of 0 . Six targets are identified on the Earth's surface, and the Coupled Selection Equations are used to deterimine which satellites should be allocated to which targets.

The equations converge quickly, and snapshots of their time evolution are shown in Fig. 2. 


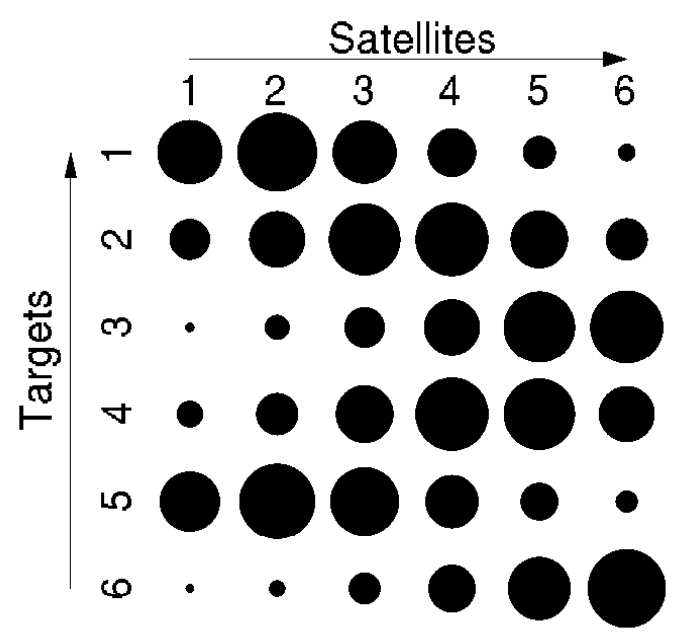

(a)

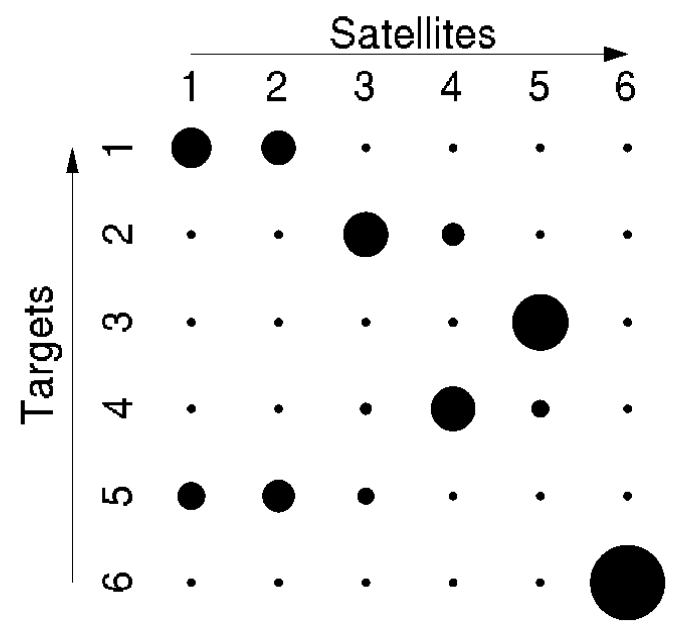

(c)

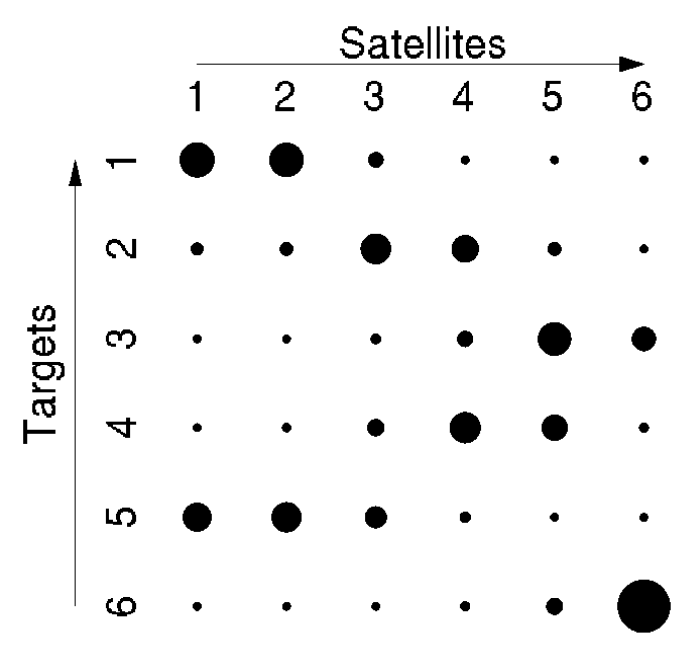

(b)

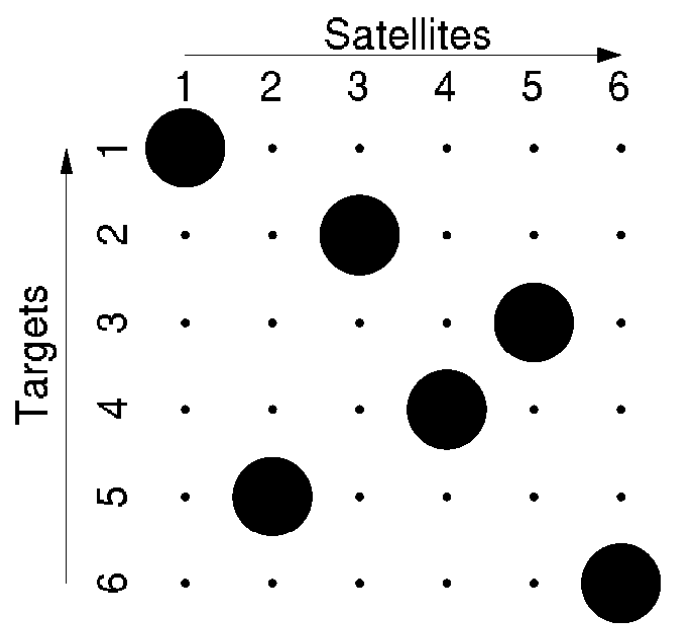

(d)

Fig. 2. Time evolution of two-index Coupled Selection Equation variables for test case \#1 - with an equal number of satellites and targets. The radii of circles represent the strength of pairing between target $i$ and satellite $j$.

As can be seen from Fig. 2, the equations converge as expected - the pairing with the highest initial strength in each row/column tends to persist and grow, whilst all others decay to zero. The only exception to this is in row 1 , where the highest initial coupling for target 1 is with satellite 2 , yet in the converged state, satellite 1 has been allocated to target 1 . This is because targets 1 and 5 both have their strongest initial couplings associated with satellite 2 . This results in a column-wise competition between the two elements via the coupling terms of the equation, and since the highest initial value of column 2 lies with target 5 , target 5 wins the competition for satellite 2. This demonstrates the subtle way that the method can deal with conflicts that arise between the satellites as they vie to be allocated to targets. The final on-orbit allocations of this simulation are displayed in Fig. 3. 


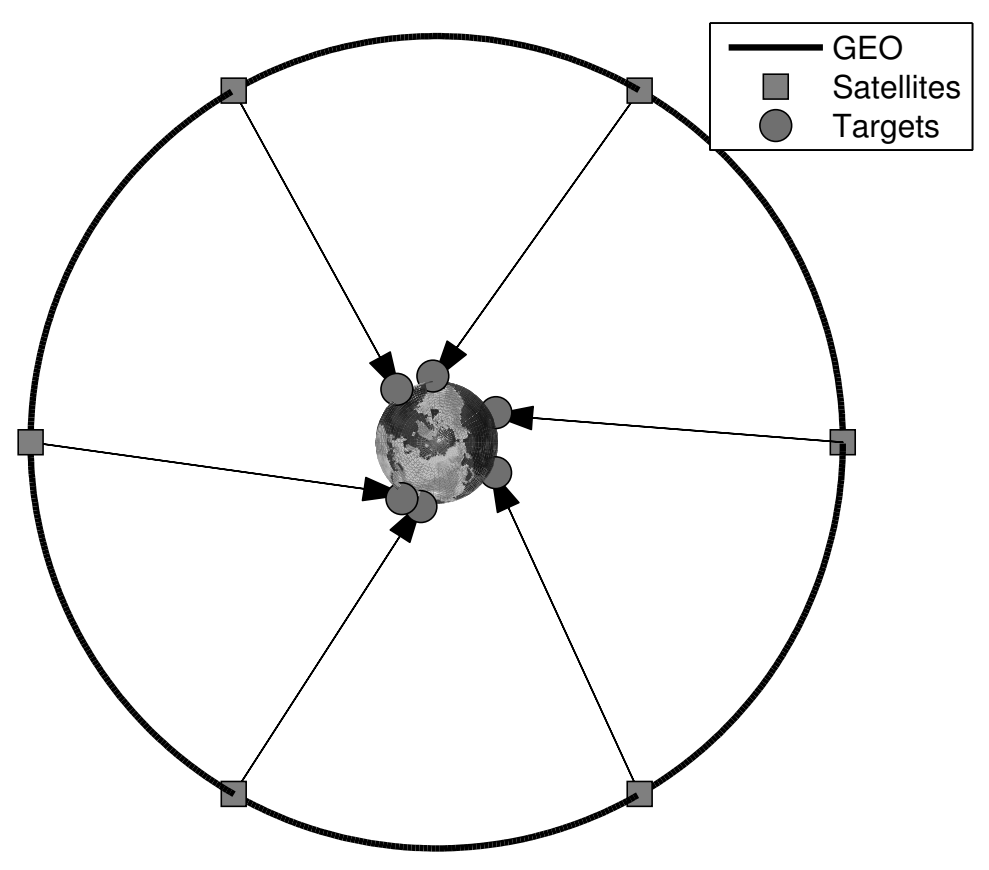

Fig. 3. Final satellite allocations for test case \#1 - with an equal number of satellites and targets.

\section{B. Demonstration of CSEs for Surplus Satellites with Satellite Failure}

In this demonstration, a constellation of seven homogenous satellites are again initially equi-spaced on geostationary orbit. Three targets are identified on the Earth's surface, and the Coupled Selection Equations are again used to deterimine which satellites should be allocated to which targets. During the process, one of the allocated satellites fails, and the coupled selection equations autonomously re-converge to allocate one of the unallocated satellites to the target of the failed satellite. Once again, snapshots of the time evolution of the equations are shown in Fig. 4.

The highest initial couplings in each row/column persist and all others decay as expected with satellites 1, 4 and 7 being allocated as shown by Fig. 4 . At $t=15 \mathrm{~s}$, a failure is triggered in satellite 1 and the unallocated satellites begin a sub-competition to fill the position of the failed satellite. This sub-competition converges as expected and satellite 2, which has the strongest coupling with the remaining target compared to the other unallocated satellites, wins the competition and fills in for failed satellite 1, as shown in Fig. 4. 

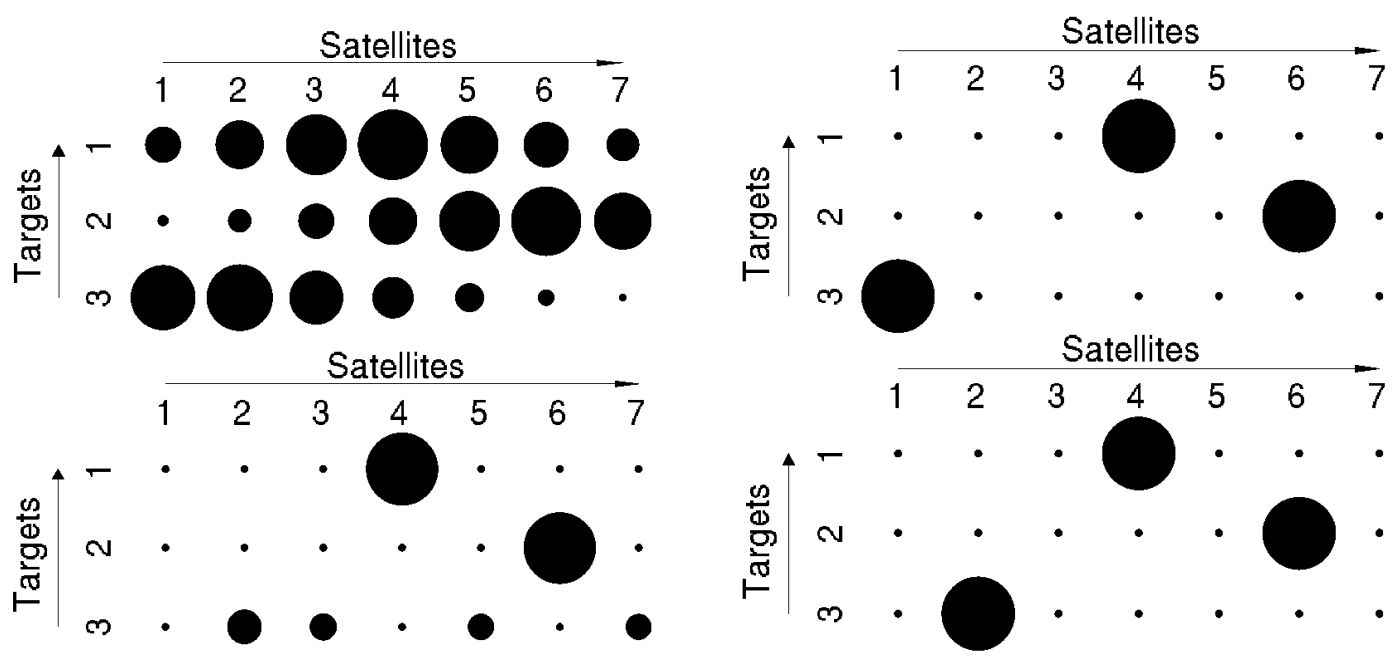

Fig. 4. Time evolution of two-index Coupled Selection Equation variables for test case \#2 - with fewer targets than available satellites. The radii of circles represent the strength of pairing between target $i$ and satellite $j$.

To provide more detail on the time evolution of the equations in this scenario, the coupled selection time histories for the pairings associated with target 3 are shown in Fig. 5.

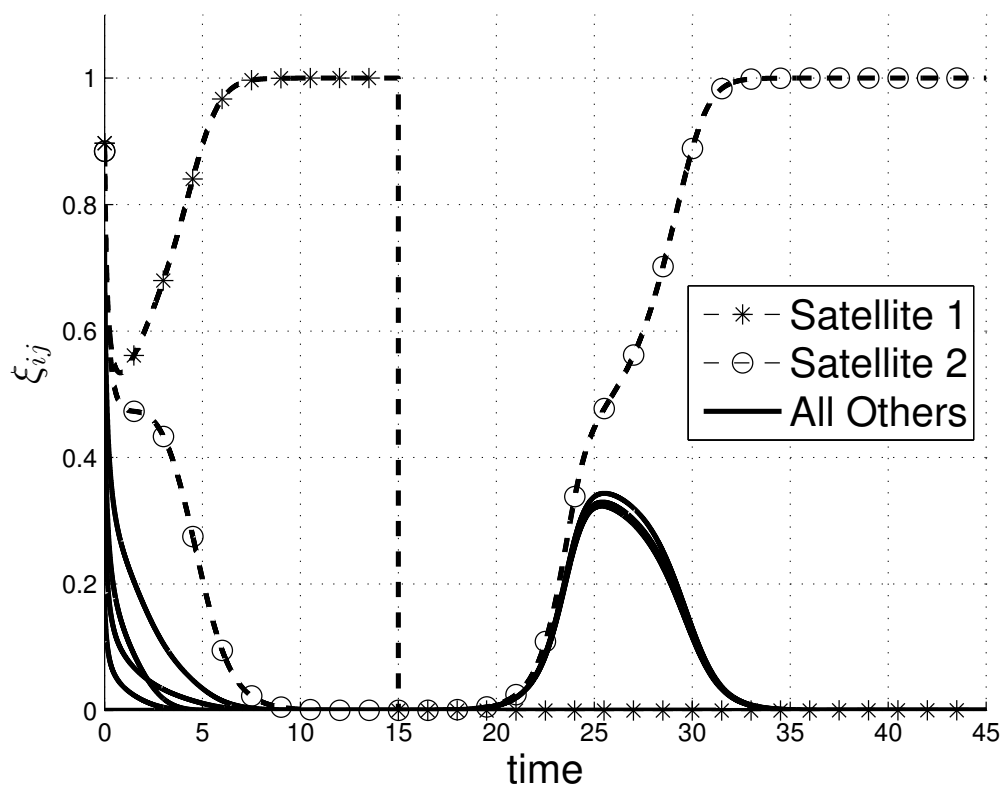

Fig. 5. Coupled Selection Equation time histories for target \#3 in test case \#2 - with fewer targets than available satellites.

Figs. 4 and 5 demonstrate how robust the Coupled Selection Equations are to agent failures, allowing quick adaptations to be made to the allocations in response to unforeseeable events.

The on-orbit allocations from before and after the failure of satellite 1 are shown in Fig 6. 

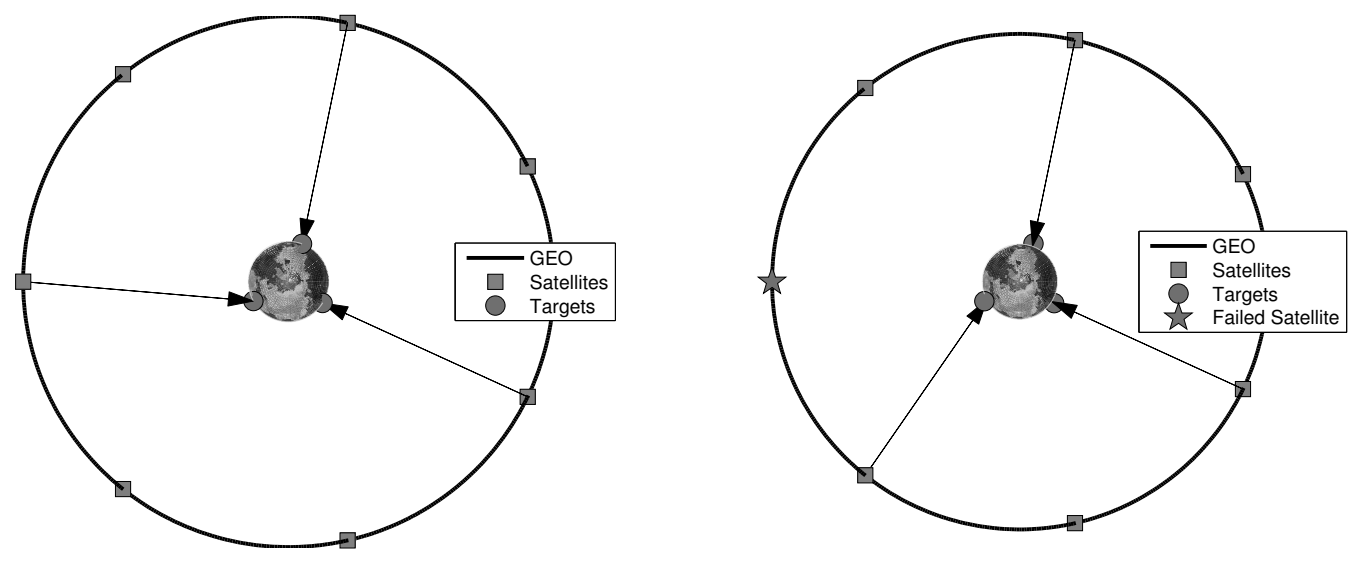

Fig. 6. Final satellite allocations for test case \#2 - with fewer targets than available satellites.

For demonstration, the same simulation was run, but with the initial $\Delta v$ s of each satellite in the cost function set such that a previously allocated satellite had a $\Delta v$ loading greater than that of the other satellites. The resulting allocations are shown in Fig. 7.

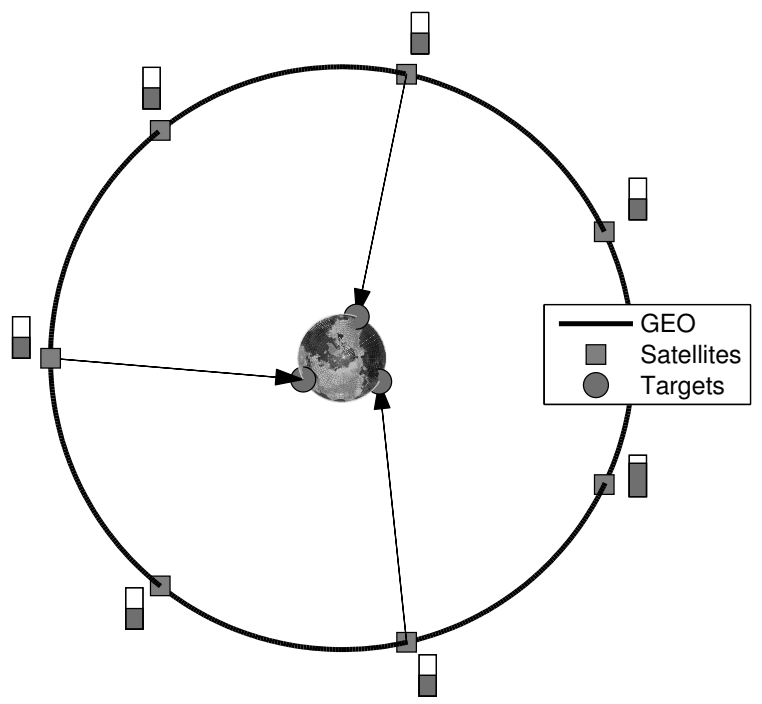

Fig. 7. Final satellite allocations for test case $\# 2$ - with an equal number of satellites and targets. In this case, the initial $\Delta v$ expenditures vary between spacecraft (shown by the bars beside each satellite), to demonstrate its effect on the allocations. A higher percentage of filled bar indicates higher previous $\Delta v$ expenditures.

As can be seen, the satellite with the highest $\Delta v$ expenditure, which had previously been allocated, has now been replaced by a satellite with a lower $\Delta v$ allocation, despite the fact that the replacement satellite is further away from the target. 


\section{Demonstration of CSEs for Multiple Satellite Types}

In this demonstration, a constellation of 6 satellites - 3 of type 1 and 3 of type 2 - are initially equi-spaced in geostationary orbit, in order to represent a disaggregated spacecraft scenario. Three targets are identified on the Earth's surface, and the Coupled Selection Equations are used to determine which two satellites of each type are allocated to which targets. The equations again converge quickly, and snapshots of their time evolution are given in Fig. 8. Here, the selection variables converge in pairs - i.e. the top (white) layer represents the possible pairs of satellites of both types that can visit target 1 , the grey layers represents the same for target 2 , etc. Only one variable in each layer should persist after convergence. The on-orbit allocations from this demonstration case are given in Fig. 9. 

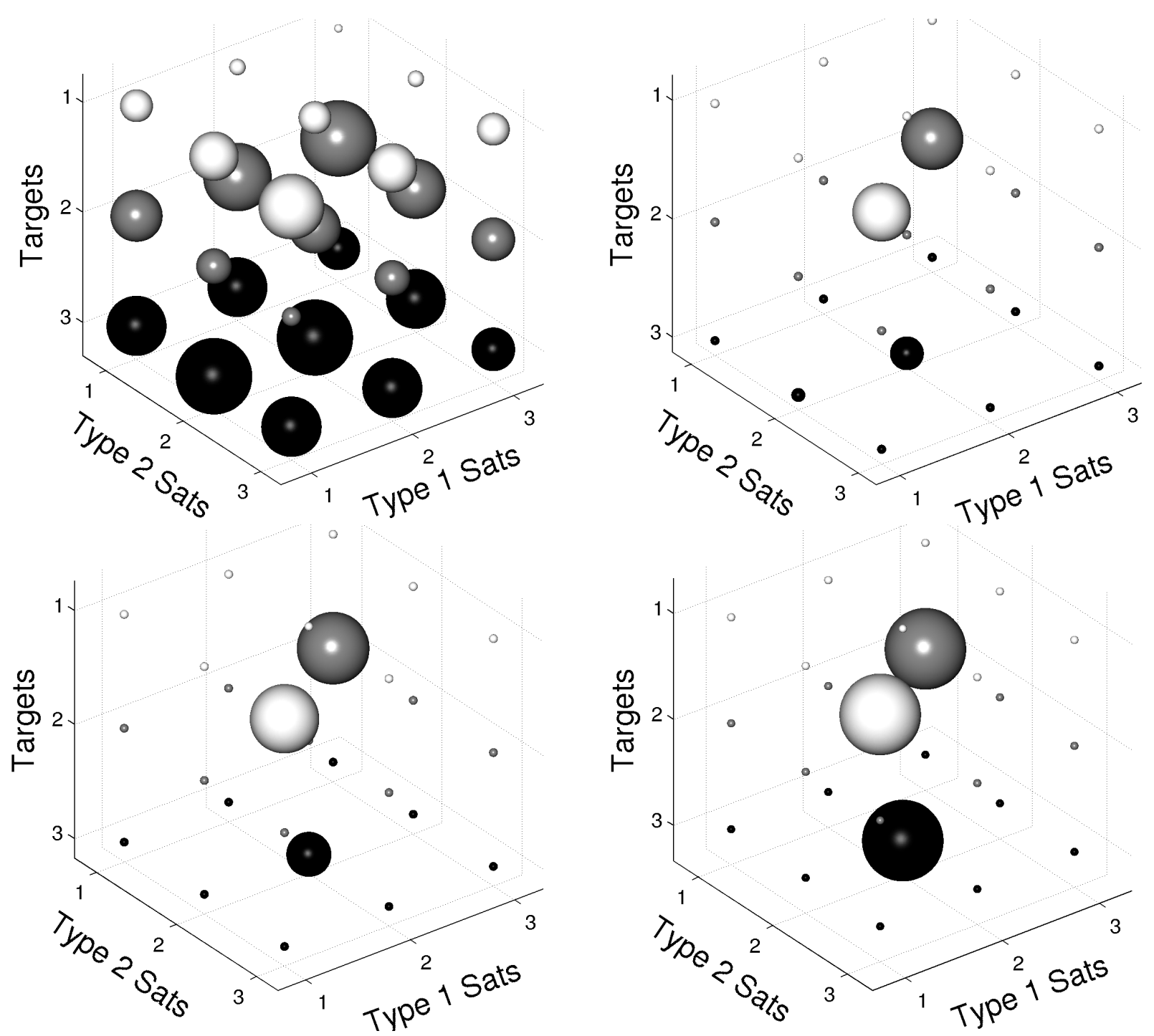

Fig. 8. Time evolutions of three-index Coupled Selection Equation variables for test case \#3 - with a fractionated satellite constellation with two types of satellites which must be allocated to targets in pairs. The radii of sphere represent the strength of pairing between target $k$ and satellite combinations $i$ and $j$. The bottom black layer represents possible pairings assigned to target 3, while the middle (grey) and top (white) layers describe the same for targets 2 and 1 respectively. 


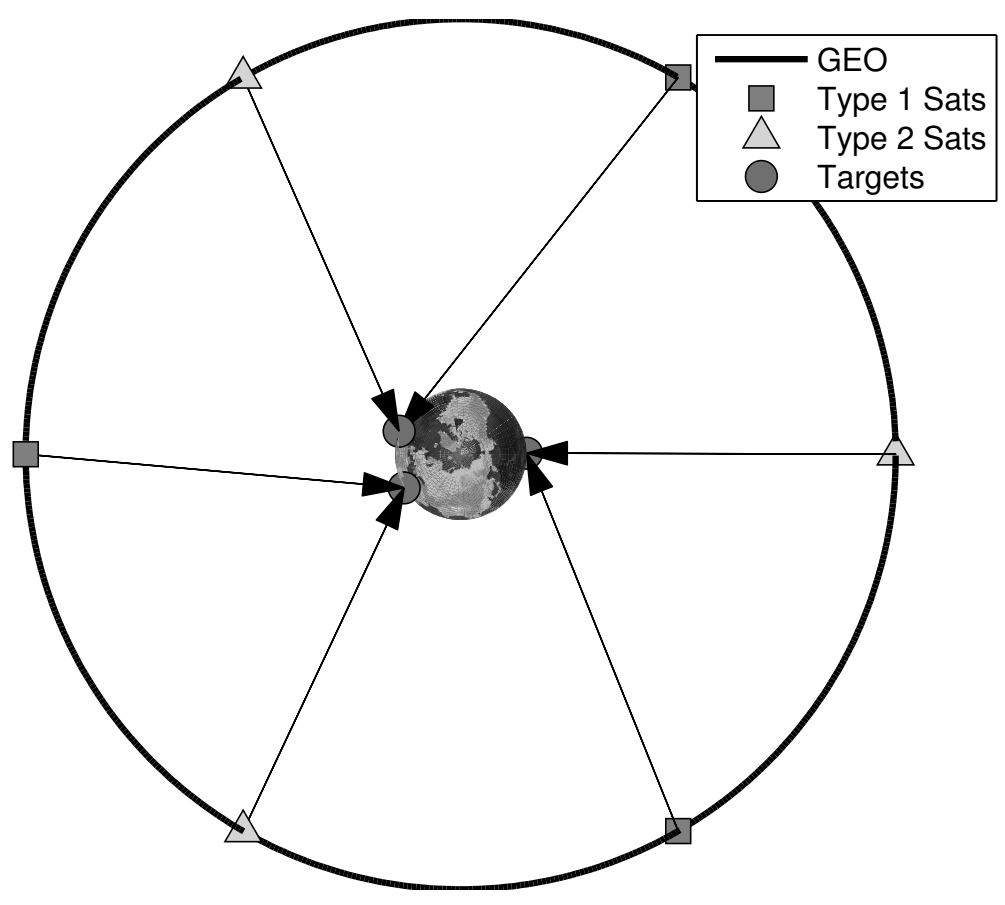

Fig. 9. Final satellite allocations for test case \#3 - - with a fractionated satellite constellation with two types of satellites which must be allocated to targets in pairs.

\section{Artificial Potential Function Actuator}

At each time step, the phase angle between each satellite and target are computed. A weighted average of the phasing angles using the coupled selection variables is then computed for each satellite according to (21).

$$
\bar{\phi}_{j}=\frac{\sum_{j=1}^{N} \xi_{i j} \phi_{i j}}{\sum_{j=1}^{N} \xi_{i j}}
$$

In the case where $\sum_{j=1}^{N}=0$ (i.e. when satellite $j$ has not been allocated to any target), then $\bar{\phi}_{j}$ is set to 0 .

The use of the weighted average ensures that the satellite maneuvers can be actuated even before the Coupled Selection Equations have fully converged. Once they have converged, the phasing angle assigned to each satellite is offset by a small amount to avoid satellite converging on precisely the same space above the target.

The desination direction, $\epsilon$, can then be calculated from (22). This variable dictates whether the satellite is leading or lagging its target, with a positive and negative values representing leads and lags respectively.

$$
\epsilon=\operatorname{sign}\left(\bar{\phi}_{j}\right)
$$

At each time-step the analytical solution in (5) is used to estimate the phase angle that the satellite would traverse if it were to return to geostationary orbit from that point, $\phi_{\mathrm{GEO}}$. This information, along with the destination direction, 
is used to raise the orbit by $r_{\text {offset }}$ when the satellite leads the target, in order to slow its angular rate with respect to the target, or to lower the orbit by $r_{\text {offset }}$ when the satellite lags the target to allow the satellite to advance upon it, as shown by (23).

$$
r_{\text {desired }}=\left\{\begin{array}{cc}
r_{\mathrm{GEO}}-r_{\mathrm{offset}} \epsilon & \left|\bar{\phi}_{j}\right|>\left|\phi_{\mathrm{GEO}}\right| \\
r_{\mathrm{GEO}} & \left|\bar{\phi}_{j}\right| \leq\left|\phi_{\mathrm{GEO}}\right|
\end{array}\right.
$$

A a simple artificial potential function controller is designed to actuate the orbit raising/lowering maneuvers. Although more sophisticated real-time guidance algorithms would be necessary for reliable real-world maneuvering, artificial potential function controllers have been used here as a simplification, in order to avoid the notoriously difficult problem of low-thrust guidance. However, the use of artificial potential function methods as a first guess approximation to autonomous guidance laws has been explored and verified by Scarrit[41]. The artificial potential function controller is constructed in order to have a minimum value corresponding to the constellation state in which the satellites are at their target orbit radii, as given in (24).

$$
V=\frac{1}{2} \sum_{j=1}^{N}\left(r_{j}-r_{\text {desired }}\right)^{2}
$$

Accordingly, the convergence of the system to its state of minimum potential, i.e. where the constellation converges upon its target orbit radii, can be assured if the rate of change of the potential function is rendered negative definite. The derivative of (24) is given by (25).

$$
\dot{V}=\sum_{j=1}^{N} \dot{r}_{j}\left(r_{j}-r_{\text {desired }}\right)
$$

Substituting (4) into (25) results in (26).

$$
\dot{V}=2 \sum_{j=1}^{N} \sqrt{\frac{r_{j}^{3}}{\mu}}\left(r_{j}-r_{\text {desired }}\right) a_{t j}
$$

It is clear from (26) that convergence will be achieved if the control acceleration is assigned according to (27).

$$
a_{t j}=-\zeta \operatorname{sgn}\left(r_{j}-r_{\text {desired }}\right)
$$

where $\zeta$ is a parameter describing the maximum acceleration magnitude available to the satellite.

This controller gives a simple thrust-coast-thrust profile, although where minimum time convergence upon the target is desired, a simple controller for continuous thrust could be implemented as an alternative [37].

The value of $\zeta$ in (27) is set such that the maximum acceleration magnitude requested by the controller does not exceed the capabilities of the on-board propulsion system. A parameter study was performed in order to understand the required values of $\zeta$ and $r_{\text {offset }}$ for different response times of the constellation, the results of which are shown in in Figs. 10 and 11. 


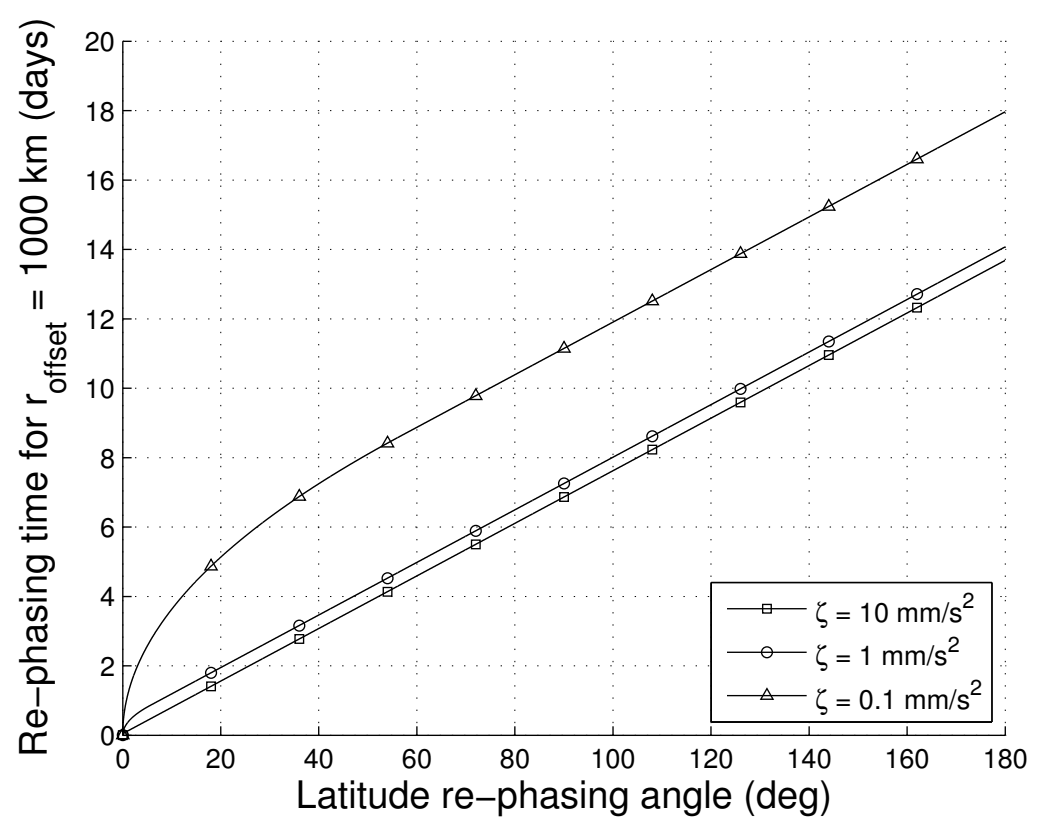

Fig. 10. Satellite rephasing time with $1000 \mathrm{~km}$ radial offset from nominal orbital altitude for varying maximum accelerations, $\zeta$.

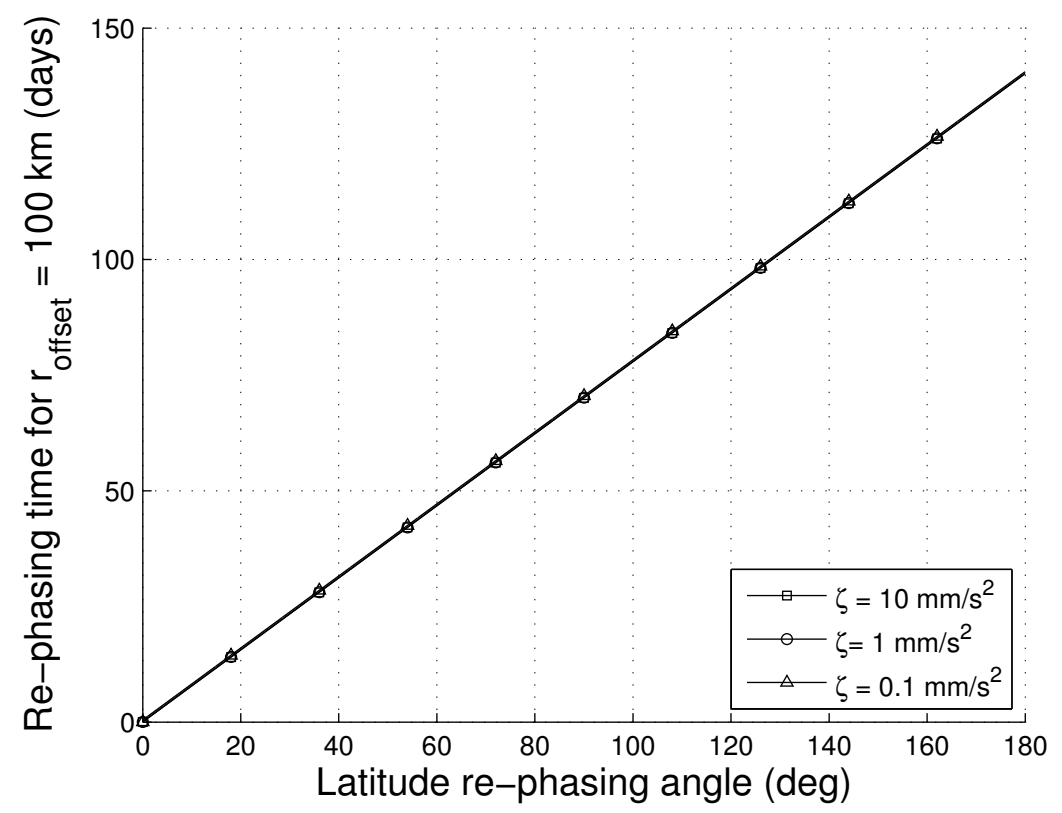

Fig. 11. Rephasing time with $100 \mathrm{~km}$ radial offset from nominal orbital altitude for varying maximum accelerations, $\zeta$.

It can be seen that the value of $\zeta$ has only a modest effect on the total re-phasing time, with almost no difference in the case where $r_{\text {offset }}=100 \mathrm{~km}$, since most of the rephasing time is spent in the coast arc. Given this, it would 
generally be best to pick as low as value as $\zeta$ as possible, as this gives the lowest $\Delta v$ expenditures, for only small increases in re-phasing times. The dominating factor with regards to the re-phasing time, then, is the radial offset. Raising the order of magnitude of $r_{\text {offset }}$ will lower the re-phasing time by approximately the same order of magnitude, but with an impact on the accumulated $\Delta v$.

Hence, for this simulation, the maximum available acceleration was set to $0.1 \mathrm{~mm} / \mathrm{s}^{2}$, equivalent to a $100 \mathrm{~kg}$ spacecraft with a $10^{-2} \mathrm{~N}$ thruster available, with $r_{\text {offset }}=1000 \mathrm{~km}$.

\section{Summary of Methodology}

The various elements implemented in this control scheme are summarised in Fig. 12, which describes the system as a simplified control block diagram.

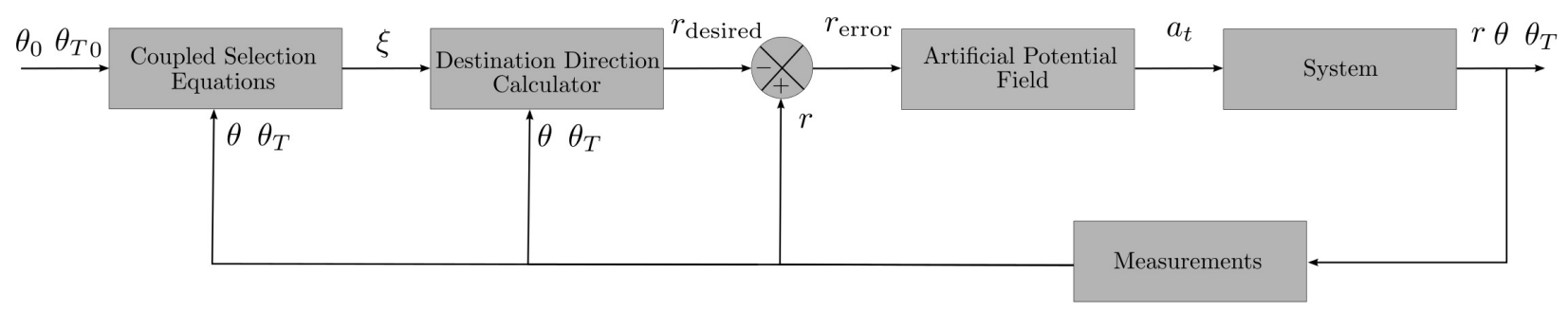

Fig. 12. Simplified control block diagram summarising the control scheme implemented where $\theta$ and $\theta_{T}$ are the angular positions of the satellites and targets respectively, $\xi$ is the output of the coupled selection equations, $r_{\text {desired }}$ are the desired orbit radii, $r_{\text {error }}$ are the orbit radii errors, $a_{t}$ is the tangential control acceleration, and $r$ are the actual orbit radii.

As can be seen from the diagram, the procedure is as follows:

- Initial angular positions of targets and satellites $\left(\theta_{0}, \theta_{T 0}\right)$ are passed to the coupled selection equations.

- Coupled selection equations calculate initial winnings, set up selection variables, $\xi$, and propagate them as described in section IV.

- Coupled selection variables are passed to destination direction calculator, together with $\theta$ and $\theta_{T}$.

- Destination direction calculator calculates whether satellites should raise or lower their orbit, depending on the their angular positions relative to the target to which they have been assigned, hence calculating $r_{\text {desired }}$.

- Desired and actual orbit radii are compared to calculate the radii error, $r_{\text {error }}$.

- $r_{\text {error }}$ is passed to the artificial potential field controller to generate tangential acceleration commands, $a_{t}$, to raise or lower orbit.

- Constellation system and earth dynamics are propagated according to system dynamics and control accelerations.

- System ouputs are measured and passed to appropriate control blocks for next control cycle. 


\section{Simulation of $\mathrm{N}=100$ Satellites}

\section{A. Demonstration of Success of Target Allocation and Maneuver}

In the final demonstration case, 100 satellites were placed in geostationary orbit, initially equally spaced. Four target scenarios were then simulated, each with varying target positions, numbers of simultaneous targets, numbers of satellites per target, and durations, as detailed in Table. I.

TABLE I

Simulation SCENARIO DETAILS.

\begin{tabular}{ccccc}
\hline \hline Scenario & Number Of Targets & $\begin{array}{c}\text { Target Longitudes } \\
(\mathrm{deg})\end{array}$ & $\begin{array}{c}\text { Number Of Satellites } \\
\text { Per Target }\end{array}$ & $\begin{array}{c}\text { Scenario Duration } \\
\text { (days) }\end{array}$ \\
\hline 1 & 2 & {$[50,120]$} & {$[50,50]$} & 20 \\
2 & 5 & {$[20,180,10,-115,-60]$} & {$[10,10,30,20,5]$} & 20 \\
3 & 3 & {$[10,45,-73]$} & {$[10,10,10]$} & 5 \\
4 & 4 & {$[20,55,-63,-25]$} & {$[10,10,10,25]$} & 20 \\
\hline \hline
\end{tabular}

The first scenario is designed to show how the constellation responds when requested to work at full capacity, i.e. when the number of satellites requested is equal to the number of satellites in the constellation. In this case, the 100 satellite constellation is required to be split between 2 targets, which may represent a situation in future telecommunications applications where two locations have an large bandwidth requirement. The second shows how the constellation deals with a relatively large number of simultaneous targets, with some satellites not being allocated. The third scenario is short in duration in order to show how the constellation responds when its target base changes mid-maneuver. In this case, an additional target is added in scenario 4 before the satellites have converged upon the 3 targets from scenario 3, which will require a re-evaluation of the allocation of satellite resources.

Figure (13) shows the satellite positions at 4 times during scenario one, as all 100 satellites converge upon 2 targets. Figure (14) shows the time histories of the satellite longitudes for scenario one. 

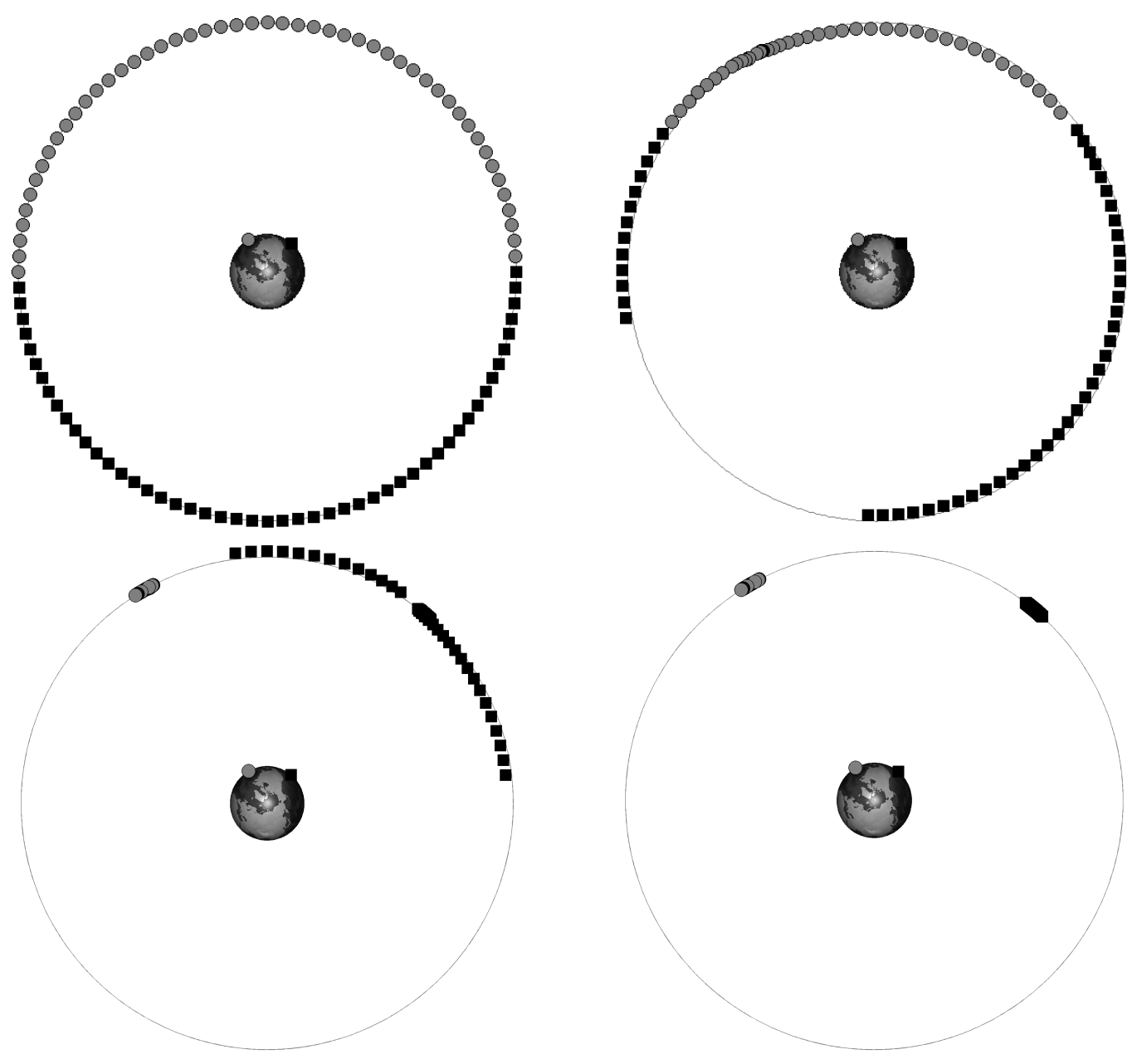

Fig. 13. Positions of satellites during scenario \#1 - with an equal number of satellites and targets. Circular markers on the Earth's surface represent the positions of targets, with the satellites colour-coded as squares to represent the allocations of satellites to targets. 


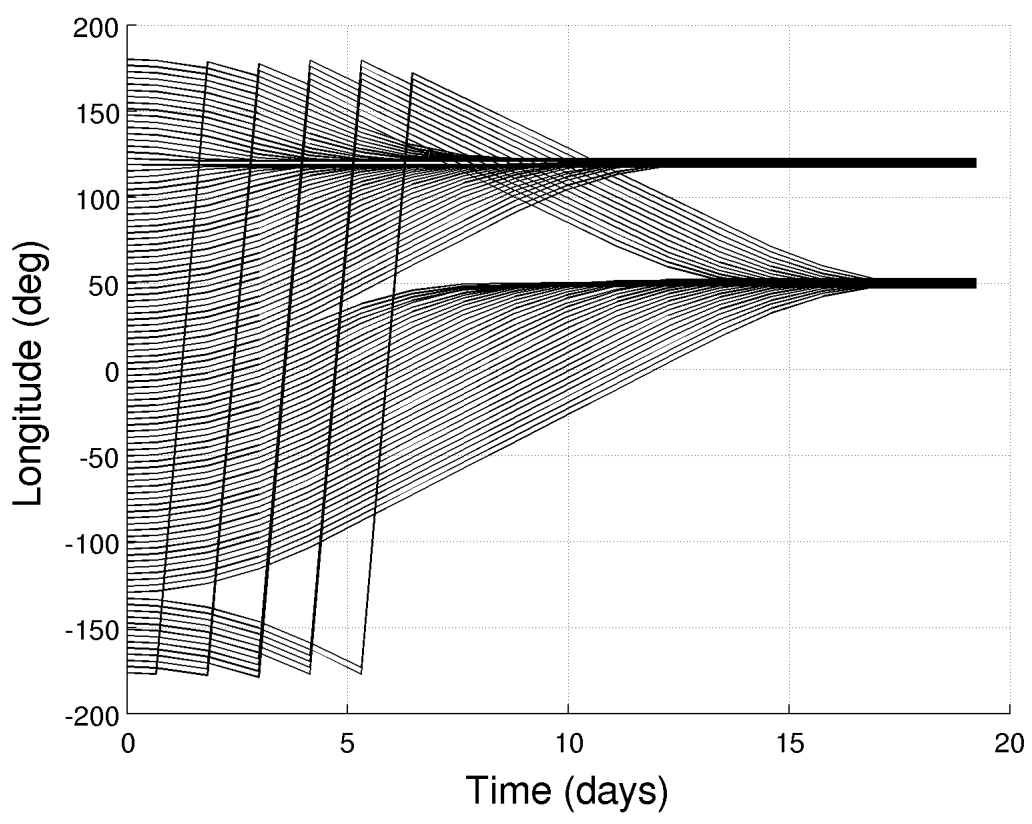

Fig. 14. Satellite longitude time histories during scenario \#1 - with an equal number of satellites and targets. All satellite longitudes can be seen converging, in equal number, to targets at 50 and 120 degrees longitude.

As can be seen from both Figs. (13) and (14), the satellites converge upon the two targets within a period of 14 days, and spread out over the target longitude.

Figure (15) shows the time histories of the satellite longitudes for scenario two. 


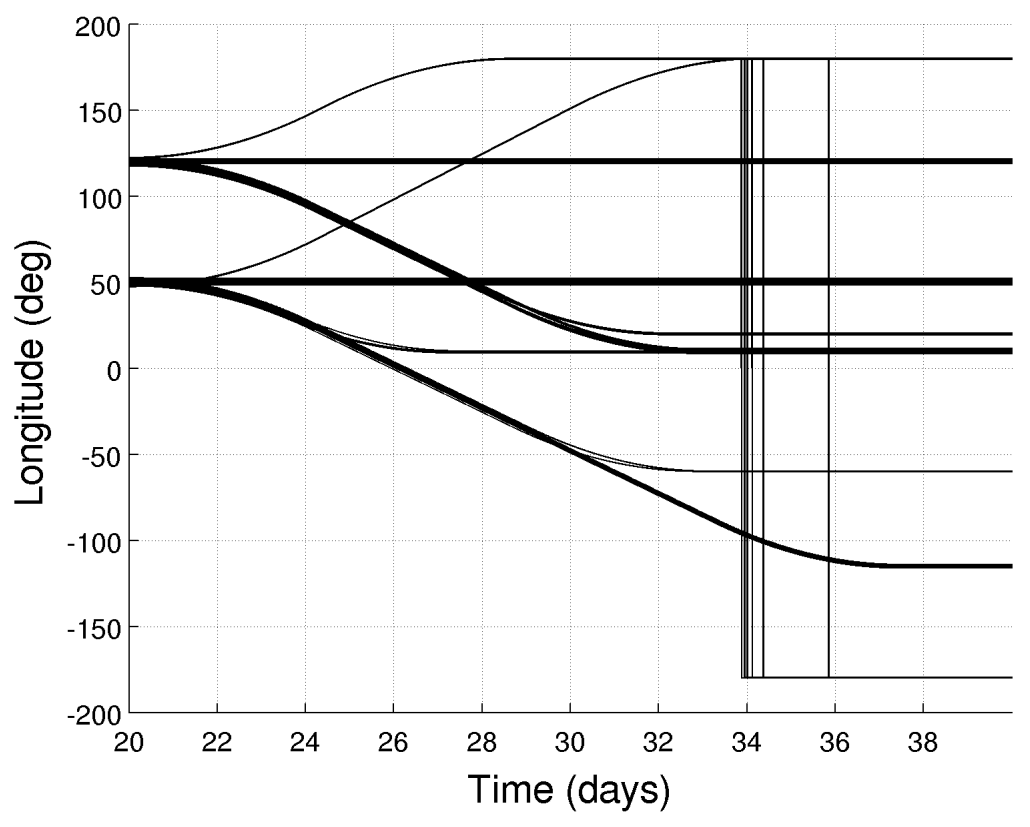

Fig. 15. Satellite longitude time histories during scenario \#2 - with fewer targets than available satellites and with a relatively large number of targets. Again, satellites can be seen converging, this time in differing number (accoring to target demand) to targets at 20, 180, 10, -115 and -60 degrees longitude. Satellites which remain static are surplus, and have not been assigned to a target.

As can be seen from Fig (15), the satellites deal well with a large number of targets, converging in just 13 days. Additionally, satellites which are not allocated remain in their previous position.

Figures (16) and (17) show the time histories of the satellite longitudes for scenarios three and four, respectively. 


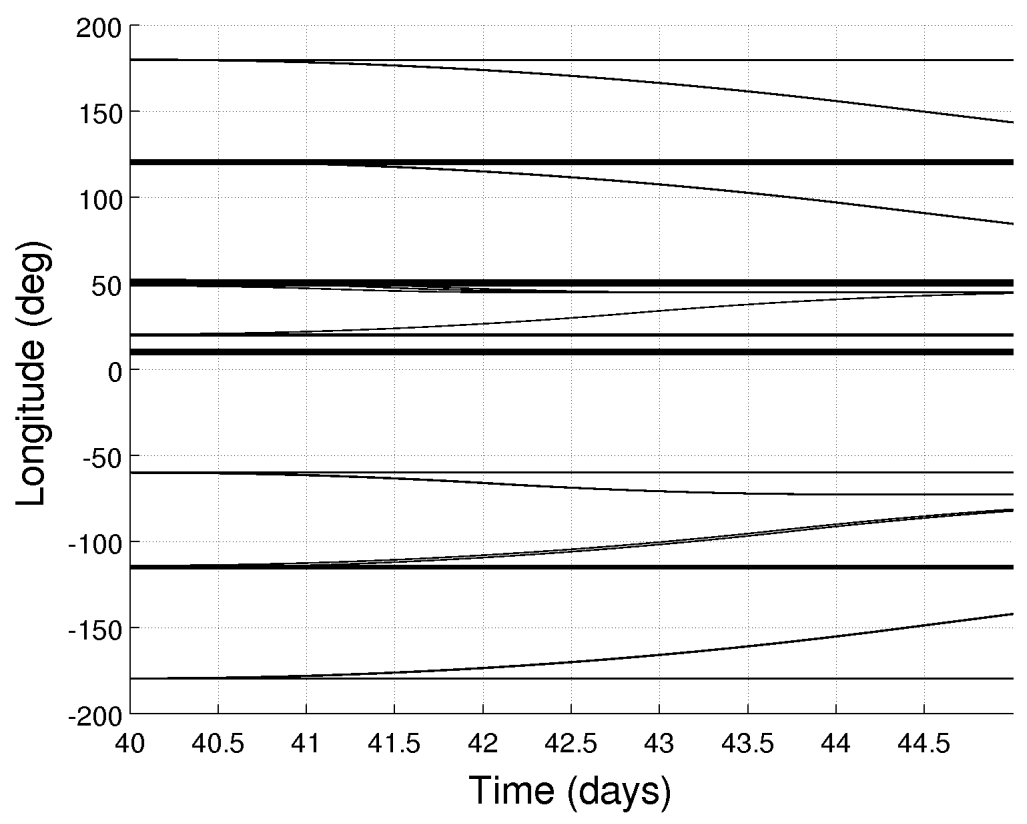

Fig. 16. Satellite longitude time histories during scenario \#3, where the targets change rapidly as the satellites are mid-manoeuvre. Again, allocated satellites can be seen beginning their manoeuvres to targets at 10, 45 and -73 degrees of longitude, but a change of target after 5 days means the satellites are reassigned before they converge. Static satellites are surplus, and not assigned to a target.

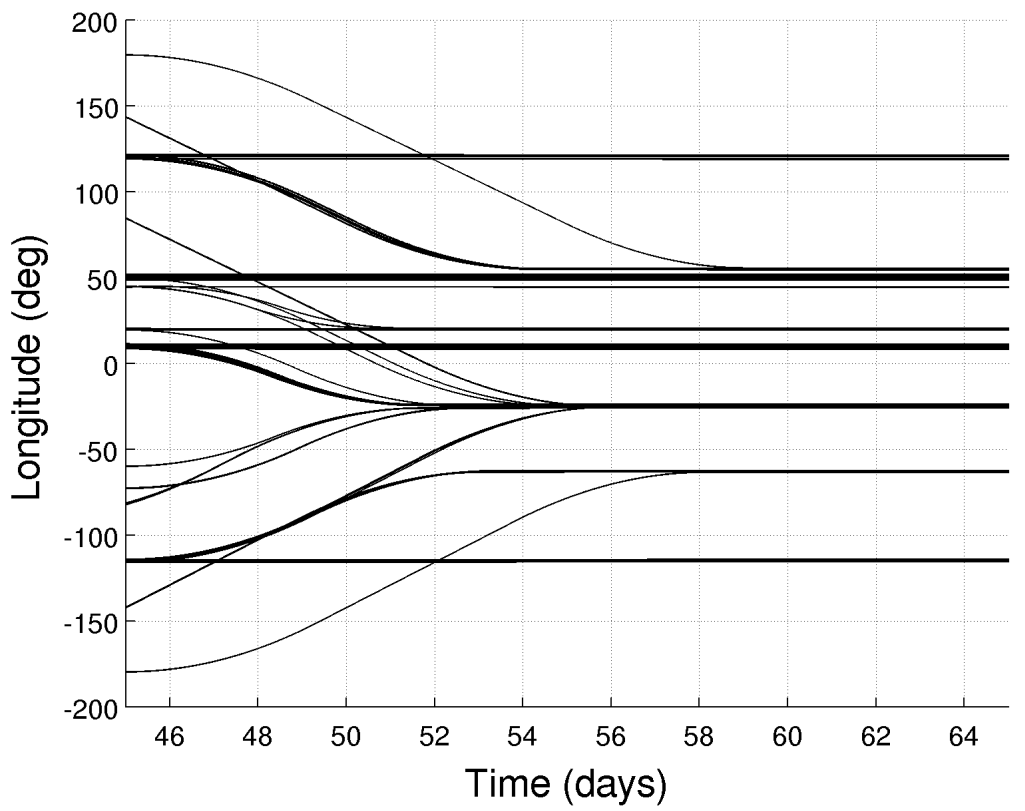

Fig. 17. Satellite longitude time histories during scenario \#4, where satellites have re-evaluated their allocations due to the change in targets during scenario \#3. Satellites can be seen converging upon targets at 20, 55, -63 and -25 degrees of longitude. Satellites with static longitudes are surplus, and not assigned to a target. 
As can be seen from Figs. (13)-(17), the satellites respond well to changes in the target positions mid-maneuver. When information about the new target is available, the coupled selection process restarts and the constellation re-evaluates its allocations. The controller is not destabilized by the sudden change in allocations.

Figure (18) shows the distribution of $\Delta v$ across the constellation after the 65 day simulation period. It shows an approximately uniform distribution, meaning that after this period, the workload is being balanced approximately uniformly across the constellation. As the constellation mission continues, the cost term in (19) associated with $\Delta v$ will grow, and thus the constellation will be forced to load balance more completely as satellite resources deplete. As is seen from the graph, the approximate $\Delta v$ expenditure for a single rephasing maneuver is in the range $[0,0.07]$ $\mathrm{km} / \mathrm{s}$.

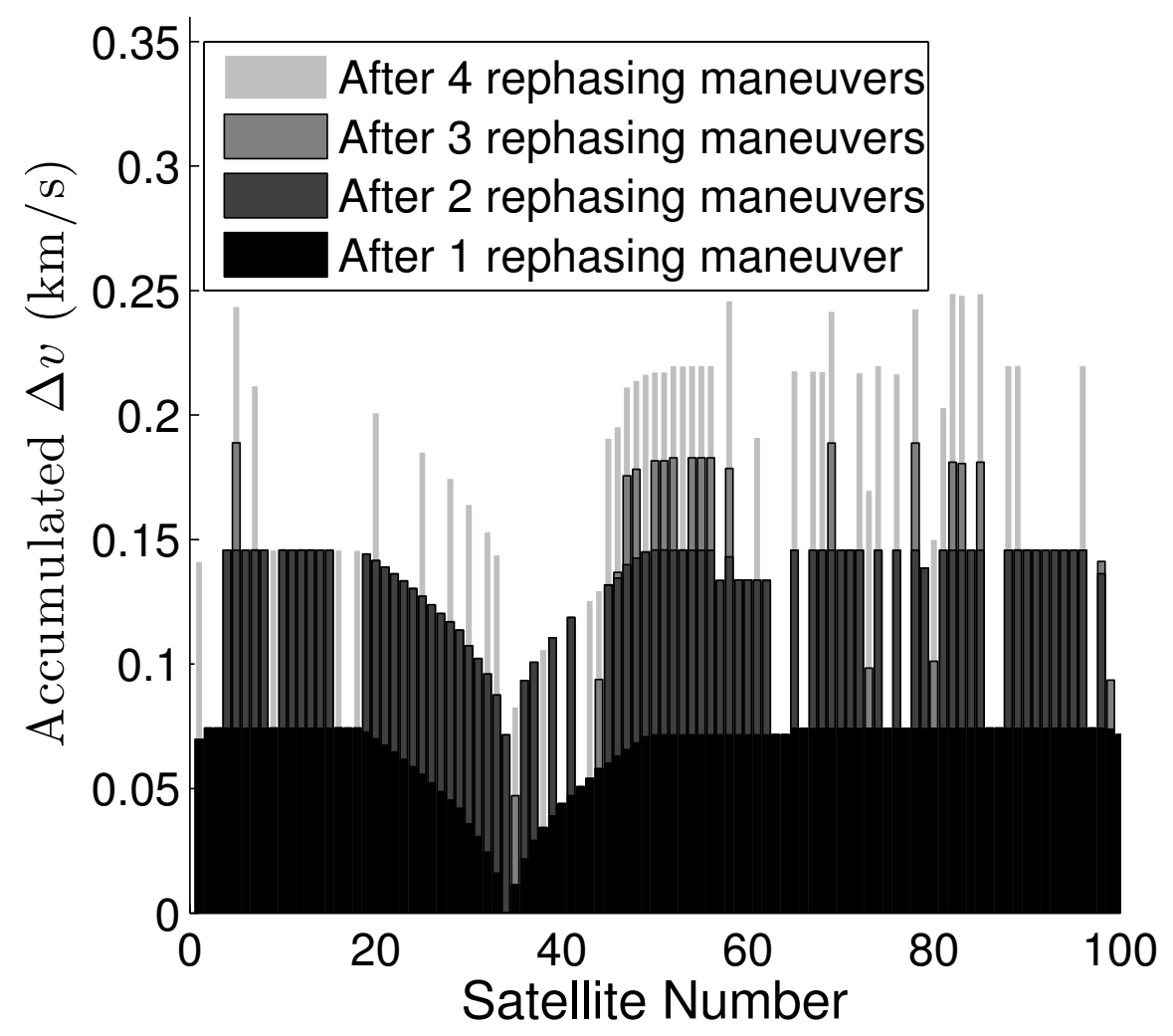

Fig. 18. Histogram of $\Delta v$ expended by constellation over the course of the 4 rephasing maneuvers simulated.

\section{CONCLUSION}

This paper has demonstrated a novel solution to the problem of autonomous task allocation on a self-organizing constellation of small satellites. Coupled Selection Equations have been shown to converge to solutions which solve the linear two-index assignment problem and axial three-index assignment problem, providing efficient pairings between satellite members and targets on the Earth's surface.

Demonstrations of the efficacy of the method have been given with various levels of complexity. Through these 
demonstrations, the method is shown to generate these solutions quickly, with very low computational effort, and is also shown to be robust to satellite failures.

In addition, a simulation with a constellation of 100 satellites has been shown, which demonstrates the applicability of the method to future swarm mission scenarios. In this simulation, the satellites respond well to the task allocation process, and take 10-20 days to arrive above their targeted longitudes in their required numbers. This time-frame is suitable for some applications, for example telecommunications and space science, where the targeted longitudes may be known in advance. However, this is not potentially quick enough for applications in, for example, disaster monitoring, which may require a faster response time. It would be possible to increase the maximum available acceleration for each spacecraft, or increase the offset in orbit radius to incur a faster relative drift, though this would lead to a reduction in the longevity of individual satellites units, increasing the frequency at which the constellation would need to be replenished. For such high accelerations, it would be necessary for the cost of replenishing the constellation to fall further, or for the propulsion technology to advance to a stage where such accelerations can be achieved from exploitation of natural orbital perturbations (i.e. solar radiation pressure), or where propulsion is near-propellantless.

In addition, the work presented here has implemented a model of the Coupled Selection Equations which is propagated on a centralised architecture. In order for the autonomy to be embedded within the constellation, the equations must be reformulated to be amenable to computation on a distributed architecture, where each agent propagates its own state using information from the constellation network. The reformulated equations must be simple enough for agents with limited processing capabilities to propagate them, and must still converge to a viable solution in the absence of information about the state of all other members of the constellation or where time delays in communications are present. This work provides a strong basis for these future developements for embedded autonomy.

Finally, this work also demonstrates the usefulness in general space-based asset allocation problems, opening up many avenues for future work and implementation. In this work, a strong emphasis is placed on algorithmic efficiency for implementation on a self-sufficient constellation of micro-satellites. However, in applications where this efficiency is not required the utility of the method in more complex and computationally demanding is vastly enhanced. This includes applications where on-ground computation with control command uplink is possible.

In addition, the method quickly and efficiently generates near-optimal solutions which would be useful as firstguess solutions for higher fidelity optimal asset allocation algorithms. 


\section{REFERENCES}

[1] D. C. Beste, "Design of satellite constellations for optimal continuous coverage," Aerospace and Electronic Systems, IEEE Transactions on, vol. AES-14, no. 3, pp. 466-473, 1978.

[2] H. Emara and C. Leondes, "Minimum number of satellites for three-dimensional continuous worldwide coverage," Aerospace and Electronic Systems, IEEE Transactions on, vol. AES-13, no. 2, pp. 108-111, 1977.

[3] R. Zandbergen, S. Dinwiddy, J. Hahn, E. Breeuwer, and D. Blonski, "Galileo orbit selection," in Proceedings of the 17th International Technical Meeting of the Satellite Division of The Institute of Navigation (ION GNSS 2004), Long Beach, CA, September 2004, pp. 616-623.

[4] R. A. Wiedeman and A. J. Viterbi, "The globalstar mobile satellite system for worldwide personal communications," in 3rd International Mobile Satellite Conference, June 1993, pp. 291-296.

[5] C. Fossa, R. Raines, G. Gunsch, and M. A. Temple, "An overview of the iridium (r) low earth orbit (leo) satellite system," in Aerospace and Electronics Conference, 1998. NAECON 1998. Proceedings of the IEEE 1998 National, Dayton, Ohio, July 1998, pp. 152-159.

[6] A. G. Y. Johnston and C. R. McInnes, "Autonomous control of a ring of satellites," in AAS/AIAA Space Flight Mechanics Meeting, no. 97-104. Huntsville, Alabama: AAS/AIAA, February 1997.

[7] X. Duan and P. Bainum, "Low-thrust autonomous control for maintaining formation and constellation orbits," in AIAA/AAS Astrodynamics Specialist Conference and Exhibit. Providence, Rhode Island: AIAA, August 2004.

[8] X. Junhua and Z. Yulin, "A coordination control method for stationkeeping of regressive orbit regional coverage satellite constellation," in Proceedings of the 25th Chinese Control Conference, Harbin, Heilongjiang, August 2006, pp. 136-139.

[9] A. Strong, "On the deployment and station keeping dynamics of n-body orbiting satellite constellations," Ph.D. dissertation, Howard University, 2000.

[10] J. Wertz, J. Collins, S. Dawson, H. Knigsmann, and C. Potterveld, "Autonomous constellation maintenance," in Mission Design and Implementation of Satellite Constellations, ser. Space Technology Proceedings, J. Ha, Ed. Springer Netherlands, 1998, vol. 1, pp. 263-273.

[11] N. H. Shah, "Automated station-keeping for satellite constellations," Master's thesis, Massachusetts Institute of Technology, June 1997.

[12] D. Krejci, B. Seifert, and C. Scharlemann, "Endurance testing of a pulsed plasma thruster for nanosatellites," Acta Astronautica, vol. 91, pp. 187-193, 2013.

[13] S. J. Pottinger, D. Krejci, and C. A. Scharlemann, "Development of a ppt for cubesat applications," in 44th AIAA/ASME/SAE/ASEE Joint Propulsion Conference and Exhibit. Hartford, CT: AIAA, July 2008.

[14] R. W. Conversano and R. E. Wirz, "Mission capability assessment of cubesats using a miniature ion thruster," Journal of Spacecraft and Rockets, vol. 50, pp. 1035-1046, 2013.

[15] S. C. Spangelo and B. W. Longmier, "Bravosat: Optimizing the delta-v capability of a cubesat mission with novel plasma propulsion technology," in Interplanetary Small satellite Conference, Pasadena, CA, June 2013.

[16] B. W. Longmier, E. A. Bering, M. D. Carter, L. D. Cassady, W. J. Chancery, and F. R. C. Diaz, "Ambipolar ion acceleration in an expanding magnetic nozzle," Plasma Sources Science and Technology, vol. 20, p. 015007, 2011.

[17] D. Kirtley and J. Fife, "Modeling, simulation, and design of an electrostatic colloid thruster," in The 29th IEEE International Conference on Plasma Science. Alberta, Canada: IEEE, May 2002.

[18] A. Huang and E.-H. Yang, "Mems thruster system for cubesat orbital maneuver," in ASME 2009 International Mechanical Engineering Congress and Exposition. Florida, USA: ASME, November 2009.

[19] B. A. Warneke and K. S. Pister, "Mems for distributed wireless sensor networks," in Proceedings of the IEEE International Conference on Electronics, Circuits, and Systems, vol. 1, Dubrovnik, Croatia, 2002, pp. 291-294.

[20] D. Barnhart, T. Vladmirova, and M. Sweeting, "Satellite-on-a-chip: A feasibility study," in Proc. 5th Round Table on Micro/Nano Technologies for Space. Nordwijk, The Netherlands: ESTEC/ESA, October 2005.

[21] D. J. Barnhart, "Very small satellite design for space sensor networks," Ph.D. dissertation, University of Surrey, Guildford, England, U.K., June 2008.

[22] D. J. Barnhart, T. Vladimirova, and M. N. Sweeting, "Very-Small-Satellite Design for Distributed Space Missions," Journal of Spacecraft and Rockets, vol. 44, pp. 1294-1306, November 2007. 
[23] J. Hu and Z. Xu, "Brief paper - distributed cooperative control for deployment and task allocation of unmanned aerial vehicle networks," Control Theory Applications, IET, vol. 7, no. 11, pp. 1574-1582, 2013.

[24] J. Bellingham, M. Tillerson, A. Richards, and J. P. How, "Multi-task allocation and path planning for cooperating uavs," in Cooperative Control: Models, Applications and Algorithms, ser. Cooperative Systems, S. Butenko, R. Murphey, and P. Pardalos, Eds. Springer US, 2003, vol. 1, pp. 23-41.

[25] C. Ze-Su, S. Li-Ning, G. Hai-Bo, and Z. Pu-Cheng, "Multi-robot cooperative pursuit based on combinatorial auction mechanism under dynamic environment," in Systems and Control in Aerospace and Astronautics, 2008. ISSCAA 2008. 2nd International Symposium on, 2008, pp. 1-6.

[26] H.-L. Choi, L. Brunet, and J. How, "Consensus-based decentralized auctions for robust task allocation," Robotics, IEEE Transactions on, vol. 25, no. 4, pp. 912-926, 2009.

[27] C.-I. Chen and V. Cherkassky, "Task allocation and reallocation for fault tolerance in multicomputer systems," Aerospace and Electronic Systems, IEEE Transactions on, vol. 30, no. 4, pp. 1094-1104, 1994.

[28] Y. Fu, Q. Ling, and Z. Tian, "Distributed sensor allocation for multi-target tracking in wireless sensor networks," Aerospace and Electronic Systems, IEEE Transactions on, vol. 48, no. 4, pp. 3538-3553, 2012.

[29] G. Radice and C. R. McInnes, "Development of a behavioural algorithm for autonomous spacecraft," in 53rd International Astronautical Congress. Houstan, Texas: IAC, October 2002.

[30] A. Badawy and C. R. McInnes, "On-orbit assembly using superquadric potential fields," Journal of Guidance, Control and Dynamics, vol. 31, no. 1, pp. 30-43, 2008.

[31] R. Lafrenz, F. Schreiber, O. Zweigle, M. Schanz, H. Rajaie, U.-P. Kappeler, P. Levi, and J. Starke, "Evaluating coupled selection equations for dynamic task assignment using a behavior framework," in Autonome Mobile Systeme 2007, ser. Informatik aktuell, K. Berns and T. Luksch, Eds. Springer Berlin Heidelberg, 2007, pp. 118-125.

[32] P. Molnar, "Self-organized navigation control for manned and unmanned vehicles in space colonies: Final report," NASA Institute of Advanced Concepts, ISRA Grant 07600-044, 2000.

[33] J. Starke, C. Ellsaesser, and T. Fukuda, "Self-organized control in cooperative robots using a pattern formation principle," Physics Letters A, vol. 375, no. 21, pp. 2094-2098, 2011.

[34] J. Starke, "Dynamical assignments of distributed autonomous robotic systems to manufacturing targets considering environmental feedbacks," in Intelligent Control, 2002. Proceedings of the 2002 IEEE International Symposium on, 2002, pp. 678-683.

[35] P. Molnar and J. Starke, "Control of distributed autonomous robotic systems using principles of pattern formation in nature and pedestrian behavior," Systems, Man, and Cybernetics, Part B: Cybernetics, IEEE Transactions on, vol. 31, no. 3, pp. 433-435, 2001.

[36] R. H. Battin, An Introduction to the Mathematics and Methods of Astrodynamics, Revised Edition. Ohio, USA: AIAA Education Series, 1999.

[37] G. Mushet, C. Colombo, and C. R. McInnes, "Autonomous control of reconfigurable constellation of satellites on geostationary orbit with artificial potential fields," in Proceedings of the 23rd International Symposium on Space Flight Dynamics, Pasadena, CA, U.S.A, October/November 2012.

[38] J. Starke and M. Schanz, Handbook of Combinatorial Optimization. Heidelberg, New York: Springer Verlag, 2012, vol. 2, ch. Dynamical System Approaches to Combinatorial Optimization, pp. 471-521.

[39] J. Starke, "Kombinatorische optimierung auf der basis gekoppelter selektionsgleichungen," Ph.D. dissertation, Universitet Stuttgart, Verlag Shaker, Aachen, 1997.

[40] B. C. Gunter and D. C. Maessen, "Space-based distributed computing using a networked constellation of small satellites," Journal of Spacecraft and Rockets, vol. 5, pp. 1086-1095, 2013.

[41] S. K. Scarrit, "A self-contained guidance and targeting algorithm for spacecraft applications," Ph.D. dissertation, The University of Texas, Austin, Texas, 2012. 


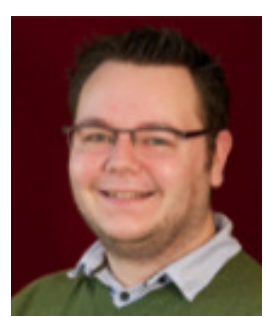

Garrie S. Mushet Garrie S. Mushet is a final year PhD student at the Advanced Space Concepts Laboratory at Strathclyde University. His work focuses on the development of new methodologies for control of the spatial pattern of massively distributed swarms/constellations of micro-spacecraft to meet various mission objectives, including earth observation, data collection for climate science, space interferometry and optimal real-time response to changes in telecommunications demand.

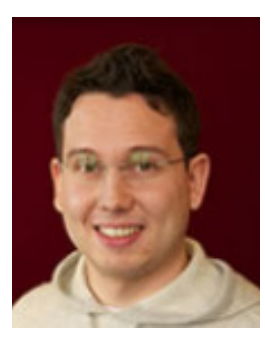

Giorgio Mingotti Dr. Giorgio Mingotti is a Research Associate at the Advanced Space Concepts Laboratory, University of Strathclyde. He is currently working under the supervision of Prof. Colin McInnes on the ERC VISIONSPACE project to investigate the orbital dynamics of micro-spacecraft. Previously, he was appointed as AstroNet-I Experienced Researcher at the Institute for Industrial Mathematics of the University of Paderborn, Germany and Research Associate at the Distributed Space Systems Laboratory, Technion - Israel Institute of Technology. Besides participating in EU-, ERC- and ESA-funded projects, he also worked as consultant engineer for Italian SMEs on aerospace and mechanical engineering projects. He holds a PhD on Trajectory Design and Optimization from Politecnico di Milano.

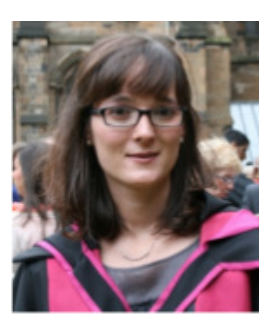

Camilla Colombo Dr. Camilla Colombo is lecturer in spacecraft engineering at the University of Southampton. She currently holds a Marie Curie Research Fellowship at Politecnico di Milano. Her research focuses on the orbital dynamics of space debris and high area-to-mass spacecraft, space trajectory design, optimisation and control, and asteroid deflection. She is currently leading and collaborating on some studies for the European Space Agency on strategies for the end-of-life disposal from highly elliptical orbits, Lagrange-point orbits, medium and low Earth orbits. Previously, she was appointed as research fellow at the Advances Space Concepts Laboratory at the University of Strathclyde and research associate at the University of Glasgow, where she completed her $\mathrm{PhD}$ and was awarded the William Wilson Scott Award Scholarship (2009).

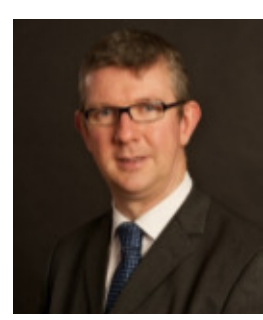

Colin R. McInnes Colin McInnes is Director of the Advanced Space Concepts Laboratory at the University of Strathclyde. His work spans highly non-Keplerian orbits, orbital dynamics and mission applications for solar sails, spacecraft control using artificial potential field methods and is reported in over 100 journal papers. Recent work is exploring new approaches to spacecraft orbital dynamics at extremes of spacecraft length-scale to underpin future space-derived products and services. McInnes has been the recipient of awards including the Royal Aeronautical Society Pardoe Space Award (2000), the Ackroyd Stuart Propulsion Prize (2003) and a Leonov medal by the International Association of Space Explorers (2007). 